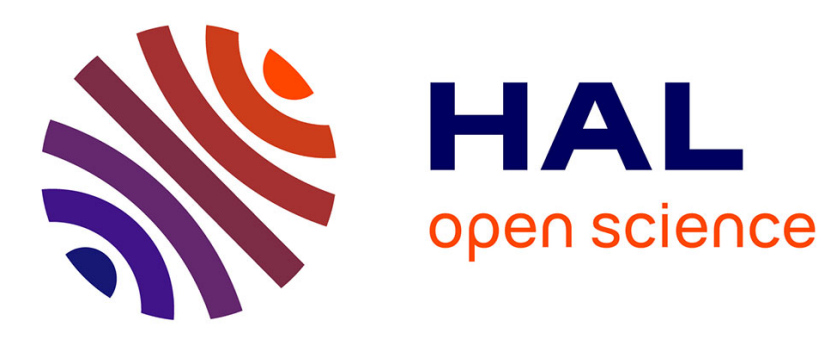

\title{
Universal Constructions that Ensure Disjoint-Access Parallelism and Wait-Freedom
}

Faith Ellen, Panagiota Fatourou, Eleftherios Kosmas, Alessia Milani, Corentin Travers

\section{- To cite this version:}

Faith Ellen, Panagiota Fatourou, Eleftherios Kosmas, Alessia Milani, Corentin Travers. Universal Constructions that Ensure Disjoint-Access Parallelism and Wait-Freedom. [Research Report] 2012. hal-00697198v2

\author{
HAL Id: hal-00697198 \\ https://hal.inria.fr/hal-00697198v2
}

Submitted on 15 May 2012

HAL is a multi-disciplinary open access archive for the deposit and dissemination of scientific research documents, whether they are published or not. The documents may come from teaching and research institutions in France or abroad, or from public or private research centers.
L'archive ouverte pluridisciplinaire HAL, est destinée au dépôt et à la diffusion de documents scientifiques de niveau recherche, publiés ou non, émanant des établissements d'enseignement et de recherche français ou étrangers, des laboratoires publics ou privés. 


\title{
Universal Constructions that Ensure Disjoint-Access Parallelism and Wait-Freedom*
}

\author{
Faith Ellen ${ }^{\ddagger}$ \\ University of Toronto \\ faith@cs.toronto.edu
}

\author{
Alessia Milani\#* \\ University of Bordeaux \\ milani@labri.fr
}

Panagiota Fatourou ${ }^{\S}$

University of Crete \& FORTH-ICS

faturu@csd.uoc.gr

\author{
Eleftherios Kosmas \\ University of Crete \& FORTH-ICS \\ ekosmas@csd.uoc.gr
}

\author{
Corentin Travers ${ }^{\dagger \dagger}$ \\ University of Bordeaux \\ travers@labri.fr
}

\begin{abstract}
Disjoint-access parallelism and wait-freedom are two desirable properties for implementations of concurrent objects. Disjoint-access parallelism guarantees that processes operating on different parts of an implemented object do not interfere with each other by accessing common base objects. Thus, disjointaccess parallel algorithms allow for increased parallelism. Wait-freedom guarantees progress for each nonfaulty process, even when other processes run at arbitrary speeds or crash.

A universal construction provides a general mechanism for obtaining a concurrent implementation of any object from its sequential code. We identify a natural property of universal constructions and prove that there is no universal construction (with this property) that ensures both disjoint-access parallelism and wait-freedom. This impossibility result also holds for transactional memory implementations that require a process to re-execute its transaction if it has been aborted and guarantee each transaction is aborted only a finite number of times.

Our proof is obtained by considering a dynamic object that can grow arbitrarily large during an execution. In contrast, we present a universal construction which produces concurrent implementations that are both wait-free and disjoint-access parallel, when applied to objects that have a bound on the number of data items accessed by each operation they support.
\end{abstract}

Topics: Distributed algorithms: design, analysis, and complexity; Shared and transactional memory, synchronization protocols, concurrent programming

Keywords: concurrent programming, disjoint-access parallelism, wait-freedom, universal construction, impossibility result

*An extending abstract of this work appeared in Proceedings of the 31st Annual ACM SIGACT-SIGOPS Symposium on Principles of Distributed Computing (PODC'12), ACM press, 2012

${ }^{\dagger}$ Supported in part by the Natural Science and Engineering Research Council of Canada.

${ }^{\ddagger}$ Some of the work was done while visiting Labri CNRS-UMR 5800, Bordeaux, France.

§Supported by the European Commission under the 7th Framework Program through the TransForm (FP7-MC-ITN-238639), Hi-PEAC2 (FP7-ICT-217068), and ENCORE (FP7-ICT-248647) projects.

"Supported by the project "IRAKLITOS II - University of Crete" of the Operational Programme for Education and Lifelong Learning 2007 - 2013 (E.P.E.D.V.M.) of the NSRF (2007 - 2013), co-funded by the European Union (European Social Fund) and National Resources.

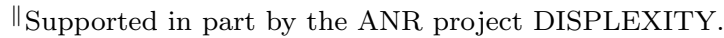

** Contact Author. Labri, University of Bordeaux 1, 351, cours de la Liberation F-33405 Talence cedex, France. Tel:+33 (0)5 400035 03. E-mail : milani@labri.fr

${ }^{\dagger}$ Supported in part by the ANR project DISPLEXITY. 


\section{Introduction}

Due to the recent proliferation of multicore machines, simplifying concurrent programming has become a necessity, to exploit their computational power. A universal construction [20] is a methodology for automatically executing pieces of sequential code in a concurrent environment, while ensuring correctness. Thus, universal constructions provide functionality similar to Transactional Memory (TM) [22]. In particular, universal constructions provide concurrent implementations of any sequential data structure: Each operation supported by the data structure is a piece of code that can be executed.

Many existing universal constructions $[1,12,15,16,19,20]$ restrict parallelism by executing each of the desired operations one after the other. We are interested in universal constructions that allow for increased parallelism by being disjoint-access parallel. Roughly speaking, an implementation is disjoint-access parallel if two processes that operate on disjoint parts of the simulated state do not interfere with each other, i.e., they do not access the same base objects. Therefore, disjoint-access parallelism allows unrelated operations to progress in parallel. We are also interested in ensuring strong progress guarantees: An implementation is wait-free if, in every execution, each (non-faulty) process completes its operation within a finite number of steps, even if other processes may fail (by crashing) or are very slow.

In this paper, we present both positive and negative results. We first identify a natural property of universal constructions and prove that designing universal constructions (with this property) which ensure both disjoint access parallelism and wait-freedom is not possible. We prove this impossibility result by considering a dynamic data structure that can grow arbitrarily large during an execution. Specifically, we consider a singly-linked unsorted list of integers that supports the operations $\operatorname{ApPEnD}(L, x)$, which appends $x$ to the end of the list $L$, and $\operatorname{SeArch}(L, x)$, which searches the list $L$ for $x$ starting from the first element of the list. We show that, in any implementation resulting from the application of a universal construction to this data structure, there is an execution of SEARCH that never terminates.

Since the publication of the original definition of disjoint-access parallelism [24], many variants have been proposed $[2,9,18]$. These definitions are usually stated in terms of a conflict graph. A conflict graph is a graph whose nodes is a set of operations in an execution. An edge exists between each pair of operations that conflict. Two operations conflict if they access the same data item. A data item is a piece of the sequential data structure that is being simulated. For instance, in the linked list implementation discussed above, a data item may be a list node or a pointer to the first or last node of the list. In a variant of this definition, an edge between conflicting operations exists only if they are concurrent. Two processes contend on a base object, if they both access this base object and one of these accesses is a non-trivial operation (i.e., it may modify the state of the object). In a disjoint-access parallel implementation, two processes performing operations $o p$ and $o p^{\prime}$ can contend on the same base object only if the conflict graph of the minimal execution interval that contains both $o p$ and $o p^{\prime}$ satisfies a certain property. Different variants of disjoint-access parallelism use different properties to restrict access to a base object by two processes performing operations. Note that any data structure in which all operations access a common data item, for example, the root of a tree, is trivially disjoint access parallel under all these definitions.

For the proof of the impossibility result, we introduce feeble disjoint-access parallelism, which is weaker than all existing disjoint-access parallelism definitions. Thus, our impossibility result still holds if we replace our disjoint-access parallelism definition with any existing definition of disjoint-access parallelism.

Next, we show how this impossibility result can be circumvented, by restricting attention to data structures whose operations can each only access a bounded number of different data items. Specifically, there is a constant $b$ such that any operation accesses at most $b$ different data items when it is applied sequentially to the data structure, starting from any (legal) state. Stacks and queues are examples of dynamic data structures that have this property. We present a universal construction that ensures wait-freedom and disjoint-access parallelism for such data structures. The resulting concurrent implementations are linearizable [23] and satisfy a much stronger disjoint-access parallelism property than we used to prove the impossibility result.

Disjoint-access parallelism and its variants were originally formalized in the context of fixed size data structures, or when the data items that each operation accesses are known when the operation starts its execution. Dealing with these cases is much simpler than considering an arbitrary dynamic data structure 
where the set of data items accessed by an operation may depend on the operations that have been previously executed and on the operations that are performed concurrently.

The universal construction presented in this paper is the first that provably ensures both wait-freedom and disjoint-access parallelism for dynamic data structures in which each operation accesses a bounded number of data items. For other dynamic data structures, our universal construction still ensures linearizability and disjoint-access parallelism. Instead of wait-freedom, it ensures that progress is non-blocking. This guarantees that, in every execution, from every (legal) state, some process finishes its operation within a finite number of steps.

\section{Related Work}

Some impossibility results, related to ours, have been provided for transactional memory algorithms. Transactional Memory (TM) [22] is a mechanism that allows a programmer of a sequential program to identify those parts of the sequential code that require synchronization as transactions. Thus, a transaction includes a sequence of operations on data items. When the transaction is being executed in a concurrent environment, these data items can be accessed by several processes simultaneously. If the transaction commits, all its changes become visible to other transactions and they appear as if they all take place at one point in time during the execution of the transaction. Otherwise, the transaction can abort and none of its changes are applied to the data items.

Universal constructions and transactional memory algorithms are closely related. They both have the same goal of simplifying parallel programming by providing mechanisms to efficiently execute sequential code in a concurrent environment. A transactional memory algorithm informs the external environment when a transaction is aborted, so it can choose whether or not to re-execute the transaction. A call to a universal construction returns only when the simulated code has been successfully applied to the simulated data structure. This is the main difference between these two paradigms. However, it is common behavior of an external environment to restart an aborted transaction until it eventually commits. Moreover, meaningful progress conditions $[11,30]$ in transactional memory require that the number of times each transaction aborts is finite. This property is similar to the wait-freedom property for universal constructions. In a recent paper [11], this property is called local progress. Our impossibility result applies to transactional memory algorithms that satisfy this progress property. Disjoint-access parallelism is defined for transactions in the same way as for universal constructions.

Strict disjoint-access parallelism [18] requires that an edge exists between two operations (or transactions) in the conflict graph of the minimal execution interval that contains both operations (transactions) if the processes performing these operations (transactions) contend on a base object. A TM algorithm is obstruction-free if a transaction can be aborted only when contention is encountered during the course of its execution. In [18], Guerraoui and Kapalka proved that no obstruction-free TM can be strictly disjoint access parallel. Obstruction-freedom is a weaker progress property than wait-freedom, so their impossibility result also applies to wait-free implementations (or implementations that ensure local progress). However, it only applies to this strict variant of disjoint-access parallelism, while we consider a much weaker disjoint-access parallelism definition. It is worth-pointing out that several obstruction-free TM algorithms [17, 21, 25, 28] satisfy a weaker version of disjoint-access parallelism than this strict variant. It is unclear whether helping, which is the major technique for achieving strong progress guarantees, can be (easily) achieved assuming strict disjoint-access parallelism. For instance, consider a scenario where transaction $T_{1}$ accesses data items $x$ and $y$, transaction $T_{2}$ accesses $x$, and $T_{3}$ accesses $y$. Since $T_{2}$ and $T_{3}$ access disjoint data items, strict disjoint-access parallelism says that they cannot contend on any common base objects. In particular, this limits the help that each of them can provide to $T_{1}$.

Bushkov et al. [11] prove that no TM algorithm (whether or not it is disjoint-access parallel) can ensure local progress. However, they prove this impossibility result under the assumption that the TM algorithm does not have access to the code of each transaction (and, as mentioned in their introduction, their impossibility result does not hold without this restriction). In their model, the TM algorithm allows the external environment to invoke actions for reading a data item, writing a data item, starting a transaction, and 
trying to commit or abort it. The TM algorithm is only aware of the sequence of invocations that have been performed. Thus, a transaction can be helped only after the TM algorithm knows the entire set of data items that the transaction should modify. However, there are TM algorithms that do allow threads to have access to the code of transactions. For instance, RobuSTM [30] is a TM algorithm in which the code of a transaction is made available to threads so that they can help one another to ensure strong progress guarantees.

Proving impossibility results in a model in which the TM algorithm does not have access to the code of transactions is usually done by considering certain high-level histories that contain only invocations and responses of high-level operations on data items (and not on the base objects that are used to implement these data items in a concurrent environment). Our model gives the universal construction access to the code of an invoked operation. Consequently, to prove our impossibility result we had to work with low-level histories, containing steps on base objects, which is technically more difficult.

Attiya et al. [9] proved that there is no disjoint-access parallel TM algorithm where read-only transactions are wait-free and invisible (i.e., they do not apply non-trivial operations on base objects). This impossibility result is proved for the variant of disjoint-access parallelism where processes executing two operations (transactions) concurrently contend on a base object only if there is a path between the two operations (transactions) in the conflict graph. We prove our lower bound for a weaker definition of disjoint-access parallelism and it applies even for implementations with visible reads. We remark that the impossibility result in [9] does not contradict our algorithm, since our implementation employs visible reads.

In [26], the concept of $M V$-permissiveness was introduced. A TM algorithm satisfies this property if a transaction aborts only when it is an update transaction that conflicts with another update transaction. An update transaction contains updates to data items. The paper [26] proved that no transactional memory algorithm satisfies both disjoint access parallelism (specifically, the variant of disjoint-access parallelism presented in [9]) and MV-permissiveness. However, the paper assumes that the TM algorithm does not have access to the code of transactions and is based on the requirement that the code for creating, reading, or writing data items terminates within a finite number of steps. This lower bound can be beaten if this requirement is violated. Attiya and Hillel [8] presented a strict disjoint-access parallel lock-based TM algorithm that satisfies MV-permissiveness.

More constraining versions of disjoint-access parallelism are used when designing algorithms [5, 6, 24]. Specifically, two operations are allowed to access the same base object if they are connected by a path of length at most $d$ in the conflict graph $[2,5,6]$. This version of disjoint-access parallelism is known as the $d$-local contention property $[2,5,6]$. The first wait-free disjoint-access parallel implementations $[24,29]$ had $O(n)$-local contention, where $n$ is the number of processes in the system, and assumed that each operation accesses a fixed set of data items. Afek et al. [2] presented a wait-free, disjoint-access parallel universal construction that has $O\left(k+\log ^{*} n\right)$-local contention, provided that each operation accesses at most $k$ predetermined memory locations. It relies heavily on knowledge of $k$. This work extends the work of Attiya and Dagan [5], who considered operations on pairs of locations, i.e. where $k=2$. Afek et al. [2] leave as an open question the problem of finding highly concurrent wait-free implementations of data structures that support operations with no bounds on the number of data items they access. In this paper, we prove that, in general, there are no solutions unless we relax some of these properties.

Attiya and Hillel [7] provide a $k$-local non-blocking implementation of $k$-read-modify-write objects. The algorithm assumes that double-compare-and-swap (DCAS) primitives are available. A DCAS atomically executes CAS on two memory words. Combining the algorithm in [7] and the non-blocking implementation of DCAS by Attiya and Dagan [5] results in a $O\left(k+\log ^{*} n\right)$-local non-blocking implementation of a $k$-readmodify-write object that only relies on single-word CAS primitives. Their algorithm can be adapted to work for operations whose data set is defined on the fly, but it only ensures that progress is non-blocking.

A number of wait-free universal constructions $[1,15,16,19,20]$ work by copying the entire data structure locally, applying the active operations sequentially on their local copy, and then changing a shared pointer to point to this copy. The resulting algorithms are not disjoint access parallel, unless vacuously so.

Anderson and Moir [3] show how to implement a $k$-word atomic CAS using LL/SC. To ensure waitfreedom, a process may help other processes after its operation has been completed, as well as during 
its execution. They employ their $k$-word CAS implementation to get a universal construction that produces wait-free implementations of multi-object operations. Both the $k$-word CAS implementation and the universal construction allow operations on different data items to proceed in parallel. However, they are not disjointaccess parallel, because some operations contend on the same base objects even if there are no (direct or transitive) conflicts between them. The helping technique that is employed by our algorithm combines and extends the helping techniques presented in [3] to achieve both wait-freedom and disjoint-access parallelism.

Anderson and Moir [4] presented another universal construction that uses indirection to avoid copying the entire data structure. They store the data structure in an array which is divided into a set of consecutive data blocks. Those blocks are addressed by a set of pointers, all stored in one LL/SC object. An adaptive version of this algorithm is presented in [15]. An algorithm is adaptive if its step complexity depends on the maximum number of active processes at each point in time, rather than on the total number $n$ of processes in the system. Neither of these universal constructions is disjoint-access parallel.

Barnes [10] presented a disjoint-access parallel universal construction, but the algorithms that result from this universal construction are only non-blocking. In Barnes' algorithm, a process $p$ executing an operation $o p$ first simulates the execution of op locally, using a local dictionary where it stores the data items accessed during the simulation of $o p$ and their new values. Once $p$ completes the local simulation of $o p$, it tries to lock the data items stored in its dictionary. The data items are locked in a specific order to avoid deadlocks. Then, $p$ applies the modifications of $o p$ to shared memory and releases the locks. A process that requires a lock which is not free, releases the locks it holds, helps the process that owns the lock to finish the operation it executes, and then re-starts its execution. To enable this helping mechanism, a process shares its dictionary immediately prior to its locking phase. The lock-free TM algorithm presented in [17] works in a similar way.

As in Barnes' algorithm, a process executing an operation op in our algorithm, first locally simulates op using a local dictionary, and then it tries to apply the changes. However, in our algorithm, a conflict between two operations can be detected during the simulation phase, so helping may occur at an earlier stage of op's execution. More advanced helping techniques are required to ensure both wait-freedom and disjoint-access parallelism.

Chuong et al. [12] presented a wait-free version of Barnes' algorithm that is not disjoint-access parallel and applies operations to the data structure one at a time. Their algorithm is transaction-friendly, i.e., it allows operations to be aborted. Helping in this algorithm is simpler than in our algorithm. Moreover, the conflict detection and resolution mechanisms employed by our algorithm are more advanced to ensure disjoint-access parallelism. The presentation of the pseudocode of our algorithm follows [12].

The first software transactional memory algorithm [27] was disjoint-access parallel, but it is only nonblocking and is restricted to transactions that access a pre-determined set of memory locations. There are other TM algorithms [14, 17, 21, 25, 28] without this restriction that are disjoint-access parallel. However, all of them satisfy weaker progress properties than wait-freedom. TL [14] ensures strict disjoint access parallelism, but it is blocking.

A hybrid approach between transactional memory and universal constructions has been presented by Crain et al. [13]. Their universal construction takes, as input, sequential code that has been appropriately annotated for processing by a TM algorithm. Each transaction is repeatedly invoked until it commits. They use a linked list to store all committed transactions. A process helping a transaction to complete scans the list to determine whether the transaction has already completed. Thus, their implementation is not disjoint-access parallel. It also assumes that no failures occur.

\section{Preliminaries}

A data structure is a sequential implementation of an abstract data type. In particular, it provides a representation for the objects specified by the abstract data type and the (sequential) code for each of the operations it supports. As an example, we will consider an unsorted singly-linked list of integers that supports the operations $\operatorname{ApPend}(v)$, which appends the element $v$ to the end of the list (by accessing a pointer end that points to the last element in the list, appending a node containing $v$ to that element, and updating the pointer to point to the newly appended node), and $\operatorname{SeARch}(v)$, which searches the list for $v$ 
starting from the first element of the list.

A data item is a piece of the representation of an object implemented by the data structure. In our example, the data items are the nodes of the singly-linked list and the pointers first and last that point to the first and the last element of the list, respectively. The state of a data structure consists of the collection of data items in the representation and a set of values, one for each of the data items. A static data item is a data item that exists in the initial state. In our example, the pointers first and last are static data items. When the data structure is dynamic, the data items accessed by an instance of an operation (in a sequential execution $\alpha$ ) may depend on the instances of operations that have been performed before it in $\alpha$. For example, the set of nodes accessed by an instance of SEARCH depends on the sequence of nodes that have been previously appended to the list.

An operation of a data structure is value oblivious if, in every (sequential) execution, the set of data items that each instance of this operation accesses in any sequence of consecutive instances of this operation does not depend on the values of the input parameters of these instances. In our example, Append is a value oblivious operation, but SEARCH is not.

We consider an asynchronous shared-memory system with $n$ processes $p_{1}, \ldots, p_{n}$ that communicate by accessing shared objects, such as registers and LL/SC objects. A register $R$ stores a value from some set and supports the operations $\operatorname{read}(R)$, which returns the value of $R$, and write $(R, v)$, which writes the value $v$ in $R$. An LL/SC object $R$ stores a value from some set and supports the operations LL, which returns the current value of $R$, and SC. By executing $\mathrm{SC}(R, v)$, a process $p_{i}$ attempts to set the value of $R$ to $v$. This update occurs only if no process has changed the value of $R$ (by executing SC) since $p_{i}$ last executed $\operatorname{LL}(R)$. If the update occurs, true is returned and we say the SC is successful; otherwise, the value of $R$ does not change and false is returned.

A universal construction provides a general mechanism to automatically execute pieces of sequential code in a concurrent environment. It supports a single operation, called PERFORM, which takes as parameters a piece of sequential code and a list of input arguments for this code. The algorithm that implements PERFORM applies a sequence of operations on shared objects provided by the system. We use the term base objects to refer to these objects and we call the operations on them primitives. A primitive is non-trivial if it may change the value of the base object; otherwise, the primitive is called trivial. To avoid ambiguities and to simplify the exposition, we require that all data items in the sequential code are only accessed via the instruction CREATEDI, READDI, and WriteDI, which create a new data item, read (any part of) the data item, and write to (any part of) the data item, respectively.

A configuration provides a global view of the system at some point in time. In an initial configuration, each process is in its initial state and each base object has its initial value. A step consists of a primitive applied to a base object by a process and may also contain local computation by that process. An execution is a (finite or infinite) sequence $C_{i}, \phi_{i}, C_{i+1}, \phi_{i+1}, \ldots, \phi_{j-1}, C_{j}$ of alternating configurations $\left(C_{k}\right)$ and steps $\left(\phi_{k}\right)$, where the application of $\phi_{k}$ to configuration $C_{k}$ results in configuration $C_{k+1}$, for each $i \leq k<j$. An execution $\alpha$ is indistinguishable from another execution $\alpha^{\prime}$ for some processes, if each of these processes takes the same steps in $\alpha$ and $\alpha^{\prime}$, and each of these steps has the same response in $\alpha$ and $\alpha^{\prime}$. An execution is solo if all its steps are taken by the same process.

From this point on, for simplicity, we use the term operation to refer to an instance of an operation. The execution interval of an operation starts with the first step of the corresponding call to PERFORM and terminates when that call returns. Two operations overlap if the call to PeRForm for one of them occurs during the execution interval of the other. If a process has invoked PERFORM for an operation that has not yet returned, we say that the operation is active. A process can have at most one active operation in any configuration. A configuration is quiescent if no operation is active in the configuration.

Let $\alpha$ be any execution. We assume that processes may experience crash failures. If a process $p$ does not fail in $\alpha$, we say that $p$ is correct in $\alpha$. Linearizability [23] ensures that, for every completed operation in $\alpha$ and some of the uncompleted operations, there is some point within the execution interval of the operation called its linearization point, such that the response returned by the operation in $\alpha$ is the same as the response it would return if all these operations were executed serially in the order determined by their linearization points. When this holds, we say that the responses of the operations are consistent. An implementation is 
linearizable if all its executions are linearizable. An implementation is wait-free [20] if, in every execution, each correct process completes each operation it performs within a finite number of steps.

Since we consider linearizable universal constructions, every quiescent configuration of an execution of a universal construction applied to a sequential data structure defines a state. This is the state of the data structure resulting from applying each operation linearized prior to this configuration, in order, starting from the initial state of the data structure.

Two operations contend on a base object $b$ if they both apply a primitive to $b$ and at least one of these primitives is non-trivial. We are now ready to present the definition of disjoint-access parallelism that we use to prove our impossibility result. It is weaker than all the variants discussed in Section 2.

Definition 1. (Feeble Disjoint-Access Parallelism). An implementation resulting from a universal construction applied to a (sequential) data structure is feebly disjoint-access parallel if, for every solo execution $\alpha_{1}$ of an operation op $p_{1}$ and every solo execution $\alpha_{2}$ of an operation op $p_{2}$, both starting from the same quiescent configuration $C$, if the sequential code of op $p_{1}$ and op $p_{2}$ access disjoint sets of data items when each is executed starting from the state of the data structure represented by configuration $C$, then $\alpha_{1}$ and $\alpha_{2}$ contend on no base objects. A universal construction is feebly disjoint-access parallel if all implementations resulting from it are feebly disjoint-access parallel.

We continue with definitions that are needed to define the version of disjoint-access parallelism ensured by our algorithm. Fix any execution $\alpha=C_{0}, \phi_{0}, C_{1}, \phi_{1}, \ldots$, produced by a linearizable universal construction $U$. Then there is some linearization of the completed operations in $\alpha$ and a subset of the uncompleted operations in $\alpha$ such that the responses of all these operations are consistent. Let op be any one of these operations, let $I_{o p}$ be its execution interval, let $C_{i}$ denote the first configuration of $I_{o p}$, and let $C_{j}$ be the first configuration at which op has been linearized. Since each process has at most one uncompleted operation in $\alpha$ and each operation is linearized within its execution interval, the set of operations linearized before $C_{i}$ is finite. For $i \leq k<j$, let $S_{k}$ denote the state of the data structure which results from applying each operation linearized in $\alpha$ prior to configuration $C_{k}$, in order, starting from the initial state of the data structure. Define $D S(o p, \alpha)$, the data set of $o p$ in $\alpha$, to be the set of all data items accessed by $o p$ when executed by itself starting from $S_{k}$, for $i \leq k<j$.

The conflict graph of an execution interval $I$ of $\alpha$ is an undirected graph, where vertices represent operations whose execution intervals overlap with $I$ and an edge connects two operations $o p$ and $o p^{\prime}$ if and only if $D S(o p, \alpha) \cap D S\left(o p^{\prime}, \alpha\right) \neq \emptyset$. The following variant of disjoint-access parallelism is ensured by our algorithm.

Definition 2. (Disjoint-Access Parallelism). An implementation resulting from a universal construction applied to a (sequential) data structure is disjoint-access parallel if, for every execution containing a process executing Perform(op $)_{1}$ and a process executing Perform $\left(o p_{2}\right)$ that contend on some base object, there is a path between $o p_{1}$ and $o p_{2}$ in the conflict graph of the minimal execution interval containing op ${ }_{1}$ and $\mathrm{op}_{2}$.

The original definition of disjoint-access parallelism in [24] differs from Definition 2 in that it does not allow two operations $o p_{1}$ and $o p_{2}$ to read the same base object even if there is no path between $o p_{1}$ and $o p_{2}$ in the conflict graph of the minimal execution interval that contains them. T Also, that definition imposes a bound on the step complexity of disjoint-access parallel algorithms. Our definition is a slightly stronger version of the disjoint-access parallel variant defined in [9] in the context of transactional memory. This definition allows two operations to contend, (but not concurrently contend) on the same base object if there is no path connecting them in the conflict graph. This definition makes the lower bound proved there stronger, whereas our definition makes the design of an algorithm (which is our goal) more difficult. Our definition is obviously weaker than strict disjoint-access parallelism [18], since our definition allows two processes to contend even if the data sets of the operations they are executing are disjoint. 


\section{Impossibility Result}

To prove the impossibility of a wait-free universal construction with feeble disjoint-access parallelism, we consider an implementation resulting from the application of an arbitrary feebly disjoint-access parallel universal construction to the singly-linked list discussed in Section 3. We show that there is an execution in which an instance of SEARCH does not terminate. The idea is that, as the process $p$ performing this instance proceeds through the list, another process, $q$, is continually appending new elements with different values. If $q$ performs each instance of APPEND before $p$ gets too close to the end of the list, disjoint-access parallelism prevents $q$ from helping $p$. This is because $q$ 's knowledge is consistent with the possibility that $p$ 's instance of SEARCH could terminate successfully before it accesses a data item accessed by $q$ 's current instance of APPEND. Also, process $p$ cannot determine which nodes were appended by process $q$ after it started the SEARCH. The proof relies on the following natural assumption about universal constructions. Roughly speaking, it formalizes that the operations of the concurrent implementation resulting from applying a universal construction to a sequential data structure should simulate the behavior of the operations of the sequential data structure.

Assumption 3 (Value-Obliviousness Assumption). If an operation of a data structure is value oblivious, then, in any implementation resulting from the application of a universal construction to this data structure, the sets of base objects read from and written to during any solo execution of a sequence of consecutive instances of this operation starting from a quiescent configuration do not depend on the values of the input parameters.

We consider executions of the implementation of a singly-linked list $L$ in which process $p$ performs a single instance of $\operatorname{SeARch}(L, 0)$ and process $q$ performs instances of $\operatorname{Append}(L, i)$, for $i \geq 1$, and possibly one instance of $\operatorname{ApPend}(L, 0)$. The sequential of the singly-linked list code is given in Appendix A. We may assume the implementation is deterministic: If it is randomized, we fix a sequence of coin tosses for each process and only consider executions using these coin tosses.

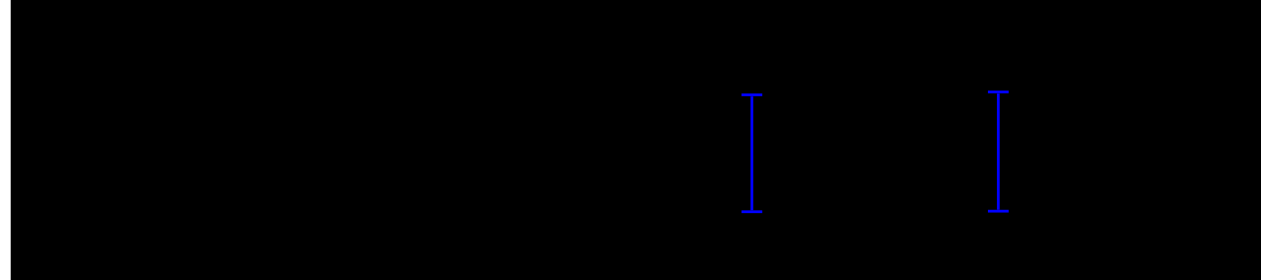

$$
\begin{aligned}
& \ldots \quad C_{i-3} \stackrel{\operatorname{APPEND}(0)}{\longrightarrow} C_{i-2}^{i-2} \stackrel{\operatorname{APPEND}(i-1)}{\longrightarrow} C_{i-1}^{i-2} \stackrel{\operatorname{APPEND}(i)}{\longrightarrow} C_{i}^{i-2} \quad \ldots
\end{aligned}
$$

Figure 1: Configurations and Sequences of Steps used in the Proof

Let $C_{0}$ be the initial configuration in which $L$ is empty. Let $\alpha$ denote the infinite solo execution by $q$ starting from $C_{0}$ in which $q$ performs $\operatorname{Append}(L, i)$ for all positive integers $i$, in increasing order. For $i \geq 1$, let $C_{i}$ be the configuration obtained when process $q$ performs $\operatorname{Append}(L, i)$ starting from configuration $C_{i-1}$. Let $\alpha_{i}$ denote the sequence of steps performed in this execution. Let $B(i)$ denote the set of base objects written to by the steps in $\alpha_{i}$ and let $A(i)$ denote the set of base objects these steps read from but do not write to. Notice that the sets $A(i)$ and $B(i)$ partition the set of base objects accessed in $\alpha_{i}$. In configuration $C_{i}$, the list $L$ consists of $i$ nodes, with values $1, \ldots, i$ in increasing order.

For $1<j \leq i$, let $C_{i}^{j}$ be the configuration obtained from configuration $C_{0}$ when process $q$ performs the first $i$ operations of execution $\alpha$, except that the $j$ 'th operation, $\operatorname{ApPend}(L, j)$, is replaced by $\operatorname{ApPend}(L, 0)$; 
namely, when $q$ performs $\operatorname{Append}(L, 1), \ldots, \operatorname{Append}(L, j-1), \operatorname{Append}(L, 0), \operatorname{Append}(L, j+1), \ldots$, $\operatorname{Append}(L, i)$. Since Append is value oblivious, the same set of base objects are written to during the executions leading to configurations $C_{i}$ and $C_{i}^{j}$. Only base objects in $\cup\{B(k) \mid j \leq k \leq i\}$ can have different values in $C_{i}$ and $C_{i}^{j}$.

For $i \geq 3$, let $\sigma_{i}$ be the steps of the solo execution of $\operatorname{SeARCH}(L, 0)$ by $p$ starting from configuration $C_{i}$. For $1<j \leq i$, let $\beta_{i}^{j}$ be the longest prefix of $\sigma_{i}$ in which $p$ does not access any base object in $\cup\{B(k) \mid k \geq j\}$ and does not write to any base object in $\cup\{A(k) \mid k \geq j\}$

Lemma 4. For $i \geq 3$ and $1<j \leq i, \beta_{i}^{j}=\beta_{i+1}^{j}$ and $\beta_{i+1}^{i-1}$ is a prefix of $\beta_{i+2}^{i}$.

Proof. Only base objects in $B(i+1)$ have different values in configurations $C_{i}$ and $C_{i+1}$. Since $\beta_{i}^{j}$ and $\beta_{i+1}^{j}$ do not access any base objects in $B(i+1)$, it follows from their definitions that $\beta_{i}^{j}=\beta_{i+1}^{j}$. In particular, $\beta_{i+2}^{i}=\beta_{i+1}^{i}$, which, by definition contains $\beta_{i+1}^{i-1}$ as a prefix.

For $i \geq 3$, let $\gamma_{i+2}$ be the (possibly empty) suffix of $\beta_{i+2}^{i}$ such that $\beta_{i+1}^{i-1} \gamma_{i+2}=\beta_{i+2}^{i}$. Figure 1 illustrates these definitions.

Let $\alpha^{\prime}=\alpha_{1} \alpha_{2} \alpha_{3} \alpha_{4} \beta_{4}^{2} \alpha_{5} \gamma_{5} \alpha_{6} \gamma_{6} \ldots$. We show that this infinite sequence of steps gives rise to an infinite valid execution starting from $C_{0}$ in which there is an instance of $\operatorname{SeARCH}(L, 0)$ that never terminates. The first steps of this execution are illustrated in Figure 2.

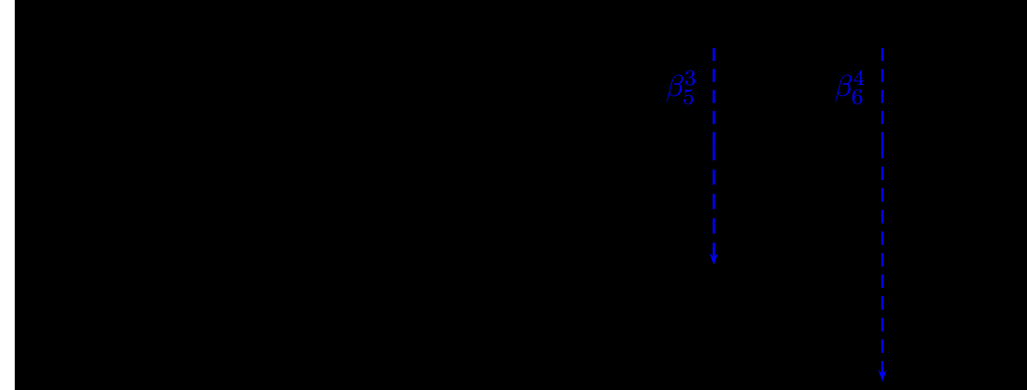

Figure 2: An Infinite Execution with a Non-terminating SEARCH Operation

Since $\beta_{4}^{2}$ does not write to any base objects accessed in $\alpha_{2} \alpha_{3} \cdots$ and, for $i \geq 4, \beta_{i+1}^{i-1}=\beta_{i}^{i-2} \gamma_{i+1}$ does not write to any base object accessed in $\alpha_{i-1} \alpha_{i} \cdots$, the executions arising from $\alpha$ and $\alpha^{\prime}$ starting from $C_{0}$ are indistinguishable to process $q$. Furthermore, since $\beta_{i+1}^{i-1}$ and, hence, $\gamma_{i+1}$ does not access any base object written to by $\alpha_{i-1} \alpha_{i} \cdots$, it follows that $\alpha_{1} \alpha_{2} \alpha_{3} \alpha_{4} \beta_{4}^{2} \alpha_{5} \gamma_{5} \cdots \alpha_{j} \gamma_{j}$ and $\alpha_{1} \alpha_{2} \alpha_{3} \alpha_{4} \cdots \alpha_{j} \beta_{j}^{j-2}$ are indistinguishable to process $p$ for all $j \geq 4$. Thus $\alpha^{\prime}$ is a valid execution.

Next, for each $i \geq 4$, we prove that there exists $j>i$ such that $\gamma_{j}$ is nonempty. By the value obliviousness assumption, only base objects in $B(i-2) \cup B(i-1) \cup B(i)$ can have different values in $C_{i}$ and $C_{i}^{i-2}$. Since $\beta_{i}^{i-2}$ does not access any of these base objects, $\beta_{i}^{i-2}$ is also a prefix of $\operatorname{SEARCH}(L, 0)$ starting from $C_{i}^{i-2}$. Since $\operatorname{SeArch}(L, 0)$ starting from $C_{i}^{i-2}$ is successful, but starting from $C_{i}$ is unsuccessful, $\operatorname{SeArch}(L, 0)$ is not completed after $\beta_{i}^{i-2}$. Therefore $\beta_{i}^{i-2}$ is a proper prefix of $\sigma_{i}$. Let $b$ be the base object accessed in the first step following $\beta_{i}^{i-2}$ in $\sigma_{i}$. For $j \geq i+1$, only base objects in $\cup\{B(k) \mid i+1 \leq k \leq j\}$ can have different values in $C_{i}$ and $C_{j}$. Therefore the first step following $\beta_{i}^{i-2}$ in $\sigma_{j}$ is the same as the first step following $\beta_{i}^{i-2}$ in $\sigma_{i}$.

To obtain a contradiction, suppose that $\beta_{i}^{i-2}=\beta_{i+3}^{i+1}$. Then $b$ is the base object accessed in the first step following $\beta_{i+3}^{i+1}$ in $\sigma_{i+3}$. By definition of $\beta_{i+3}^{i+1}$, there is some $\ell \geq i+1$ such that the first step following $\beta_{i+3}^{i+1}$ in $\sigma_{i+3}$ is either an access to $b \in B(\ell)$ or a write to $b \in A(\ell)$.

Let $S$ denote the state of the data structure in configuration $C_{\ell-1}^{\ell-3}$. In state $S$, the list has $\ell-1$ nodes and the third last node has value 0 . Thus, the set of data items accessed by $\operatorname{SEARCH}(L, 0)$ starting from state $S$ consists of L.first and the first $\ell-3$ nodes of the list. This is disjoint from the set of data items 
accessed by $\operatorname{Append}(L, \ell)$ starting from state $S$, which consists of L.last, the last node of the list, and the newly appended node. Hence, by feeble disjoint access parallelism, the solo executions of $\operatorname{Append}(L, \ell)$ and $\operatorname{SeARCH}(L, 0)$ starting from $C_{\ell-1}^{\ell-3}$ contend on no base objects.

By the value obliviousness assumption, $B(\ell)$ is the set of base objects written to in the solo execution of $\operatorname{Append}(L, \ell)$ starting from $C_{\ell-1}^{\ell-3}$ and $A(\ell)$ is the set of base objects read from, but not written to in that execution.

By the value obliviousness assumption, only base objects in $B(\ell-3) \cup B(\ell-2) \cup B(\ell-1)$ can have different values in $C_{\ell-1}$ and $C_{\ell-1}^{\ell-3}$. Since $\beta_{i}^{i-2}$ does not access any of these base objects, $\beta_{i}^{i-2}$ is also a prefix of $\operatorname{SeARCH}(L, 0)$ starting from $C_{\ell-1}^{\ell-3}$ and the first step following $\beta_{i}^{i-2}$ in this execution is the same as the first step following $\beta_{i}^{i-2}$ in $\sigma_{i}$. Recall that this is either an access to $b \in B(\ell)$ or a write to $b \in A(\ell)$. Thus, the solo executions of $\operatorname{Append}(L, \ell)$ and $\operatorname{SeARch}(L, 0)$ starting from $C_{\ell-1}^{\ell-3}$ contend on $b$. This is a contradiction. Hence, $\beta_{i}^{i-2} \neq \beta_{i+3}^{i+1}$ and it follows that at least one of $\gamma_{i+1}, \gamma_{i+2}$, and $\gamma_{i+3}$ is nonempty.

Therefore $\gamma_{j}$ is nonempty for infinitely many numbers $j$ and, in the infinite execution $\alpha^{\prime}$, process $p$ never completes its operation $\operatorname{SEARCH}(L, 0)$, despite taking an infinite number of steps. Hence, the implementation is not wait-free and we have proved the following result:

Theorem 5. No feebly disjoint-access parallel universal construction is wait-free.

\section{The DAP-UC Algorithm}

To execute an operation $o p$, a process $p$ locally simulates the execution of $o p$ 's instructions without modifying the shared representation of the simulated state. This part of the execution is the simulation phase of $o p$. Specifically, each time $p$ accesses a data item while simulating $o p$, it stores a copy in a local dictionary. All subsequent accesses by $p$ to this data item (during the same simulation phase of $o p$ ) are performed on this local copy. Once all instructions of $o p$ have been locally simulated, op enters its modifying phase. At that time, one of the local dictionaries of the helpers of $o p$ becomes shared. All helpers of $o p$ then use this dictionary and apply the modifications listed in it. In this way, all helpers of $o p$ apply the same updates for $o p$, and consistency is guaranteed.

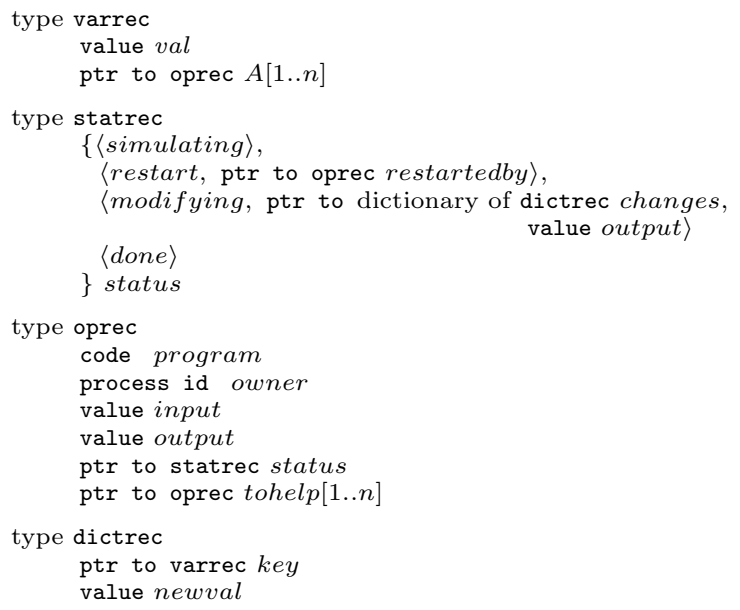

Figure 3: Type definitions

The algorithm maintains a record for each data item $x$. The first time op accesses $x$, it makes an announcement by writing appropriate information in $x$ 's record. It also detects conflicts with other operations that are accessing $x$ by reading this record. So, conflicts are detected without violating disjoint access parallelism. The algorithm uses a simple priority scheme, based on the process identifiers of the owners of 
the operations, to resolve conflicts among processes. When an operation op determines a conflict with an operation $o p^{\prime}$ of higher priority, op helps $o p^{\prime}$ to complete before it continues its execution. Otherwise, op causes $o p^{\prime}$ to restart and the owner of $o p$ will help $o p^{\prime}$ to complete once it finishes with the execution of $o p$, before it starts the execution of a new operation. The algorithm also ensures that before $o p^{\prime}$ restarts its simulation phase, it will help op to complete. These actions guarantee that processes never starve.

We continue with the details of the algorithm. The algorithm maintains a record of type oprec (lines 11-17) that stores information for each initiated operation. When a process $p$ wants to execute an operation $o p$, it starts by creating a new oprec for $o p$ and initializing it appropriately (line 22). In particular, this record provides a pointer to the code of $o p$, its input parameters, its output, the status of $o p$, and an array indicating whether $o p$ should help other operations after its completion and before it returns. We call $p$ the owner of op. To execute op, $p$ calls Help (line 23). To ensure wait-freedom, before $o p$ returns, it helps all other operations listed in the tohelp array of its oprec record (lines 24-25). These are operations with which op had a conflict during the course of its execution, so disjoint-access parallelism is not violated. The algorithm also maintains a record of type varrec (lines 1-3) for each data item $x$, This record contains a val field, which is an LL/SC object that stores the value of $x$, and an array $A$ of $n$ LL/SC objects, indexed by process identifiers, which stores oprec records of operations that are accessing $x$. This array is used by operations to announce that they access $x$ and to determine conflicts with other operations that are also accessing $x$.

The execution of $o p$ is done in a sequence of one or more simulation phases (lines 34-53) followed by a modification phase (lines 54-62). In a simulation phase, the instructions of op are read (lines 36, 37, and 50 ) and the execution of each one of them is simulated locally. The first time each process $q$ helping $o p$ (including its owner) needs to access a data item (lines 38, 43), it creates a local copy of it in its (local) dictionary (lines 42,46 ). All subsequent accesses by $q$ to this data item (during the current simulation phase of $o p$ ) are performed on this local copy (line 48). During the modification phase, $q$ makes the updates of $o p$ visible by applying them to the shared memory (lines 56-62).

The status field of $o p$ determines the execution phase of op. It contains a pointer to a record of type statrec (lines 4-10) where the status of $o p$ is recorded. The status of op can be either simulating, indicating that $o p$ is in its simulation phase, modifying, if $o p$ is in its modifying phase, done, if the execution of $o p$ has been completed but op has not yet returned, or restart, if op has experienced a conflict and should re-execute its simulation phase from the beginning. Depending on which of these values status contains, it may additionally store another pointer or a value.

To ensure consistency, each time a data item $x$ is accessed for the first time, $q$ checks, before reading the value of $x$, whether $o p$ conflicts with other operations accessing $x$. This is done as follows: $q$ announces $o p$ to $x$ by storing a pointer opr to op's oprec in $A[o p r \rightarrow o w n e r]$. This is performed by calling AnNOUnCE (line 39). ANNOUNCE first performs an LL on $\operatorname{var}_{x} \rightarrow A[p]$ (line 68), where $v_{a r}$ is the varrec for $x$ and $p=$ opr $\rightarrow$ owner. Then, it checks if the status of op (line 69) remains simulating and, if this is so, it performs an SC to store $o p$ in $v a r_{x} \rightarrow A[p]$ (line 70). These instructions are then executed one more time. This is needed because an obsolete helper of an operation, initiated by $p$ before $o p$, may successfully execute an SC on $\operatorname{var}_{x} \rightarrow A[p]$ that stores a pointer to this operation's oprec. However, we prove in Section 6 that this can happen only once, so executing the instructions on lines 68-70 twice is enough to ensure consistency.

After announcing $o p$ to $v a r_{x}, q$ calls ConfliCTs (line 40) to detect conflicts with other operations that access $x$. In Conflicts, $q$ reads the rest of the elements of $v^{2} r_{x} \rightarrow A$ (lines 76-77). Whenever a conflict is detected (i.e., the condition of the if statement of line 78 evaluates to true) between $o p$ and some other operation $o p^{\prime}$, Conflicts first checks if $o p^{\prime}$ is in its modifying phase (line 82) and, if so, it helps $o p^{\prime}$ to complete. In this way, it is ensured that, once an operation enters its modification phase, it will complete its operation successfully. Therefore, once the status of an operation becomes modifying, it will next become done, and then, henceforth, never change. If the status of $o p^{\prime}$ is simulating, $q$ determines which of $o p$ or $o p^{\prime}$ has the higher priority (line 84). If $o p^{\prime}$ has higher priority (line 89), then $o p$ helps $o p^{\prime}$ by calling HeLP $\left(o p^{\prime}\right)$. Otherwise, $q$ first adds a pointer $o p r^{\prime}$ to the oprec of $o p^{\prime}$ into opr $\rightarrow$ tohelp (line 85), so that the owner of $o p$ will help $o p^{\prime}$ to complete after $o p$ has completed. Then $q$ attempts to notify $o p^{\prime}$ to restart, using SC (line 87 ) to change the status of $o p^{\prime}$ to restart. A pointer opr is also stored in the status field of $o p^{\prime}$. When $o p^{\prime}$ 
value PERFORM(prog, input) by process $p$ :

opptr $:=$ pointer to a new oprec record

opptr $\rightarrow$ program $:=$ prog, opptr $\rightarrow$ input $:=$ input, opptr $\rightarrow$ output $:=\perp$

opptr $\rightarrow$ owner $:=$ p, opptr $\rightarrow$ status $:=$ simulating, opptr $\rightarrow$ tophelp $[1 . . n]:=[$ nil,$\ldots$, nil $]$

HELP (opptr)

/ $^{*}$ helps its own operation */

for $p^{\prime}:=1$ to $n$ excluding $p$ do /* $p$ helps operations that have been restarted by its operation op */

if $\left(\right.$ opptr $\rightarrow$ tohel $p\left[p^{\prime}\right] \neq$ nil $)$ then HeLP $\left(\right.$ opptr $\rightarrow$ tohelp $\left.\left[p^{\prime}\right]\right)$

return $($ opptr $\rightarrow$ output $)$

HeLP (opptr) by process $p$ :

opstatus $:=\mathrm{LL}($ opptr $\rightarrow$ status $)$

while (opstatus $\neq$ done)

if opstatus $=\left\langle\right.$ restart, opptr $\left.{ }^{\prime}\right\rangle$ then

$\operatorname{HELP}\left(o p p t r^{\prime}\right)$

$\mathrm{SC}($ opptr $\rightarrow$ status, $\langle$ simulating $\rangle)$

/* $o p^{\prime}$ has restarted $o p * /$ opstatus $:=\operatorname{LL}($ opptr $\rightarrow$ status $)$ /* first help $o p^{\prime} *$ /

if opstatus $=\langle$ simulating $\rangle$ then

dict $:=$ pointer to a new empty dictionary of dictrec records

${ }^{*}$ try to change the status of op back to simulating */

ins $:=$ the first instruction in opptr $\rightarrow$ program

while ins $\neq \operatorname{return}(v)$

if ins is (Writedi $(x, v)$ or $\operatorname{ReadDI}(x))$ and (there is no dictrec with key $x$ in dict)

then

ANNOUNCE $($ opptr, $x)$

CONFLICTS $($ opptr,$x)$

if ins $=\operatorname{READDI}(x)$ then $\operatorname{val}_{x}:=x \rightarrow$ val else $v_{a l}:=v$

add new dictrec $\left\langle x, v^{2} l_{x}\right\rangle$ to dict

else if ins is CREATEDI() then

$x:=$ pointer to a new varrec record

$x \rightarrow A[1 . . n]:=[$ nil $, \ldots, n i l]$

add new dictrec $\langle x$, nil $\rangle$ to dict

else

* ins is $\operatorname{WriteDI}(x, v)$ or $\operatorname{ReadDI}(x)$ and there is a dictrec with key $x$ in dict *

$/ *$ or ins is not a Writedi(), ReadDi() or Createdi() instruction */ execute ins, using/changing the value in the appropriate entry of dict if necessary

if $\neg \mathrm{VL}($ opptr $\rightarrow$ status $)$ then break

ins $:=$ next instruction of opptr $\rightarrow$ program

$/ *$ end while $* /$

if ins is $\operatorname{return}(v)$ then

$\mathrm{SC}($ opptr $\rightarrow$ status, $\langle$ modifying, dict,$v\rangle)$ opstatus $:=\mathrm{LL}($ opptr $\rightarrow$ status $)$

if opstatus $=\langle$ modifying, changes, out $\rangle$ then

opptr $\rightarrow$ outputs $:=$ out

for each dictrec $\langle x, v\rangle$ in the dictionary pointed to by changes do

$\mathrm{LL}(x \rightarrow$ val $)$

if $\neg \mathrm{VL}($ opptr $\rightarrow$ status $)$ then return

$\mathrm{SC}(x \rightarrow$ val, $v)$

$\mathrm{LL}(x \rightarrow$ val $)$

if $\neg \mathrm{VL}($ opptr $\rightarrow$ status $)$ then return

$\mathrm{SC}(x \rightarrow$ val, $v)$

${ }^{*}$ end for $* /$

$\mathrm{SC}($ opptr $\rightarrow$ status, done $)$

/* end while*/

opstatus $:=\mathrm{LL}($ opptr $\rightarrow$ status $)$

${ }^{*}$ end of the simulation of ins */

/* $v$ may be empty */ $/^{*}$ try to change status of $o p$ to modifying $* /$
not changed since beginning of simulation $* /$ access of $x$ by this attempt of op $* /$
$*$ announce that op is accessing $x * /$ $/ *$ possibly, help or restart other operations accessing $x * /$
$x:=v \quad / *$ ins is a write to $x$ of $v * /$
$/ *$ create a local copy of $x * /$ $/ *$ possibly, help or restart other operations accessing $x * /$
$x:=v \quad / *$ ins is a write to $x$ of $v * /$
$/ *$ create a local copy of $x * /$

/* start a new simulation phase */ /* to store the values of the data items */

* simulate instruction ins of $o p * /$ 
restarts its simulation phase, it will help op to complete (lines 30-33), if op is still in its simulation phase, before it continues with the re-execution of the simulation phase of $o p^{\prime}$. This guarantees that $o p$ will not cause $o p^{\prime}$ to restart again.

Recall that each helper $q$ of $o p$ maintains a local dictionary. This dictionary contains an element of type dictrec (lines 18-20) for each data item that $q$ accesses (while simulating $o p$ ). A dictionary element corresponding to data item $x$ consists of two fields, key, which is a pointer to $v a r_{x}$, and newval, which stores the value that $o p$ currently knows for $x$. Notice that only one helper of $o p$ will succeed in executing the SC on line 52, which changes the status of op to modifying. This helper records a pointer to the dictionary it maintains for $o p$, as well as its output value, in op's status, to make them public. During the modification phase, each helper $q$ of $o p$ traverses this dictionary, which is recorded in the status of op (lines 54, 56). For each element in the dictionary, it tries to write the new value into the varrec of the corresponding data item (lines 57-59). This is performed twice to avoid problems with obsolete helpers in a similar way as in ANNOUNCE.

Theorem 6. The DAP-UC universal construction (Figures 3 and 4) produces disjoint-access parallel, waitfree, concurrent implementations when applied to objects that have a bound on the number of data items accessed by each operation they support.

\section{Proof of the DAP-UC Algorithm}

\subsection{Preliminaries}

The proof is divided in three parts, namely consistency (Section 6.2), wait-freedom (Section 6.3) and disjointaccess parallelism (Section 6.4). The proof considers an execution $\alpha$ of the universal construction applied to some sequential data structure. The configurations referred to in the proof are implicitly defined in the context of this execution. We first introduce a few definitions and establish some basic properties that follow from inspection of the code.

Observe that an oprec is created only when a process begins PERForm (on line 22). Thus, we will not distinguish between an operation and its oprec.

Observation 7. The status of each oprec is initially simulating (line 22). It can only change from simulating to modifying (lines 34,52), from modifying to done (lines 54,63), from simulating to restart (lines 83,87), and from restart to simulating (lines 30,32).

Thus, once the status of an oprec becomes modifying, it can only change to done.

Observation 8. Let op be any operation and let opptr be the pointer to its oprec. When a process returns from $\operatorname{HeLP}($ opptr) (on line 58, 61 or 65), opptr $\rightarrow$ status $=$ done.

This follows from the exit condition of the while loop (line 29) and the fact that, once the status of an oprec becomes modifying, it can only change to done.

Observation 9. In every configuration, there is at most one oprec owned by each process whose status is not done.

This follows from the fact that, when a process returns from PERFORM (on line 26), has also returned from a call to HELP (on line 23), so the status of the oprec it created (on line 22) has status done, and the fact that a process does not call PERFORM recursively, either directly or indirectly.

Observation 10. For every varrec, $A[i], 1 \leq i \leq n$, is initially nil and is only changed to point to oprecs with owner $i$.

This follows from the fact that $A[i], 1 \leq i \leq n$, is initialized to nil when the varrec is created (on line 44) and is updated only on lines 70 or 73 . 


\subsection{Consistency}

An attempt is an endeavour by a process to simulate an operation. Formally, let $o p$ be any operation initiated by process $q$ in $\alpha$ and let opptr be the pointer to its oprec, i.e., opptr $\rightarrow$ owner $=q$.

Definition 11. An attempt of op by a process $p$ is the longest execution interval that begins when $p$ performs $a$ LL on opptr $\rightarrow$ status on line 28, 33, or 53 that returns simulating and during which opptr $\rightarrow$ status does not change.

The first step after the beginning of an attempt is to create an empty dictionary of dictrecs (line 35). So, each dictionary is uniquely associated with an attempt. We say that an attempt is active at each configuration $C$ contained in the execution interval that defines the attempt.

Let $p$ be a process executing an attempt att of op. If immediately after the completion of $a t t, p$ successfully changes opptr $\rightarrow$ status to $\langle$ modifying, chgs, val $\rangle$ (by performing a SC on opptr $\rightarrow$ status on line 52), then att is successful. Notice that, in this case, chgs is a pointer to the dictionary associated with att.

By Observation 7, only one process executing an attempt of op can succeed in executing the SC that changes the status of op to $\langle$ modifying, , , $\rangle$ (on line 52). Next observation then follows from the definition of a successful attempt:

Observation 12. For each operation, there is at most one successful attempt.

In att, $p$ simulates instructions on behalf of op (lines $34-52$ ). The simulation of an instruction ins starts when ins is fetched from op's program (on lines 36 or 50) and ends either just before the next instruction starts simulated, or just after the execution of the SC on line 52 if ins is the last instruction of opptr $\rightarrow$ program.

When $p$ simulates a CREATEDI() instruction, it allocates a new varrec record $x$ in its own stripe of shared memory (line 44) and adds a pointer to it in the dictionary associated with att (line 46); in this case, we also say that $p$ simulates the creation of, or creates $x$. Notice that $x$ is initially private, as it is known only by $p$; it may later become public if att is successful. Next definition captures precisely the notion of public varrec.

We say that a varrec $x$ is referenced by operation op in some configuration $C$, if opptr $\rightarrow$ status $=$ $\langle$ modifying, chgs, _- $\rangle$, where chgs is a pointer to a dictionary that contains a dictrec record whose first component, key, is a pointer to $x$.

Definition 13. $A$ varrec $x$ is public in configuration $C$ if and only if it is static or there exists an operation that references $x$ in $C$ or in some configuration that precedes it.

We say that $p$ simulates an access of (or access) some varrec $x$ by (for) $o p$, if it either simulates an ins $\in\left\{\operatorname{ReADDI}(x), \operatorname{WriteDI}\left(x,{ }_{-}\right)\right\}$, or creates $x$. Observe that if $x$ is public in configuration $C$, it is also public in every configuration that follows. Also, before it is made public, $x$ cannot be accessed by a process that has not created it.

Observation 14. If, in att, $p$ starts the simulation of an instruction ins $\in\left\{\mathrm{WRITEDI}\left(x,{ }_{-}\right), \operatorname{READDI}(x)\right\}$ at some configuration $C$, then either $x$ is created by $p$ in att before $C$, or there exists a configuration preceding the simulation of ins in which $x$ is public.

Notice that each time $p$ accesses for the first time a varrec $x$ during att, a new dictrec record is added for $x$ to the dictionary associated with att (on lines 42 or 46). From this and by inspecting the code lines $38,42,43$ and 46 follows the observation bellow.

Observation 15. If $a$ varrec $x$ is accessed by $p$ during att for op, then the first time that it accesses $x$, the following hold:

1. $p$ executes either lines 38 to 42 or lines 43 to 46 exactly once for $x$,

2. $p$ inserts a dictrec record for $x$ in the dictionary associated with att exactly once, i.e., this record is unique. 
We say that $p$ announces op on a varrec $x$ during att, if it successfully executes an SC of line 70 or line 73 on $x . A[q]$ (recall that opptr $\rightarrow$ owner $=q$ ) with value opptr, during a call of ANNOUnCE(opptr, $x$ ) (on line 39). Distinct processes may perform attempts of the same operation op. However, once an operation has been announced to a varrec, it can only be replaced by a more recent operation owned by the same process (i.e., one initiated by $q$ after $o p$ 's response), as shown by the next lemma.

Lemma 16. Assume that $p$ calls Announce $(o p p t r, x)$ in att. Suppose that in the configuration $C_{A}$ imme- $^{-}$ diately after $p$ returns from that call, att is active. Then, in configuration $C_{A}$ and every configuration that follows in which oppptr $\rightarrow$ status $\neq$ done,$(x . A[$ opptr $\rightarrow$ owner $])=$ opptr.

Proof. Since att is active when $p$ returns from Announce(opptr, $x)$, the tests performed on lines 69 and 72 are successful. So, $p$ performed LL $(x \rightarrow A[q]$, opptr $)$ on lines 68 and 71 respectively. Let $C_{L L 1}$ and $C_{L L 2}$ be the configurations immediately after $p$ performed line 68 and 71, respectively.

Let $C$ be a configuration after $p$ has returned from the call of Announce $(o p p t r, x)$ in which opptr $\rightarrow$ status $\neq$ done. Assume, by contradiction, that $(x . A[q])=$ opptr $^{\prime}$ in $C$, where opptr' is a pointer to an operation $o p^{\prime} \neq o p$. Let $p^{\prime}$ be the last process that changes the value of $x . A[q]$ to opptr' before $C$. Therefore $p^{\prime}$ performed a successful SC $\left(x . A[q], o p p t r^{\prime}\right)$ on line 70 or line 73 . This SC is preceded by a VL(opptr ${ }^{\prime} \rightarrow$ status) (on line 69 or line 72 ), which is itself preceded by a $\mathrm{LL}\left(x \rightarrow A[q]\right.$ ) (on line 68 or line 71 ). Denote by $C_{S C}^{\prime}, C_{V L}^{\prime}$ and $C_{L L}^{\prime}$, respectively, the configurations that immediately follow each of these steps. Since the VL applied by $p^{\prime}$ on (opptr $\rightarrow$ status) is successful, opptr $r^{\prime} \rightarrow$ status $=$ simulating in configuration $C_{V L}^{\prime}$.

By Observation 10, opptr $\rightarrow$ owner $=q$. By Observation 9, in every configuration, there is only one operation owned by $q$ whose status is not done. Since $o p$ has status simulating when $p$ started its attempt and the status of $o p$ is not equal to done in $C$, it then follows from Observation 7 that the status of $o p^{\prime}$ is done when the attempt att of $o p$ by $p$ started. Therefore, configuration $C_{V L}^{\prime}$, in which the status of $o p^{\prime}$ is simulating, must precede the first configuration in which att is active. In particular, $C_{V L}^{\prime}$ precedes $C_{L L 1}$ and thus $C_{L L}^{\prime}$ precedes $C_{L L 1}$.

We consider two cases according to the order in which $C_{L L 2}$ and $C_{S C}^{\prime}$ occur:

- $C_{S C}^{\prime}$ occurs before $C_{L L 2}$. In that case, no process performs a successful $\mathrm{SC}\left(x \rightarrow A[q]\right.$, opptr $\left.{ }^{\prime \prime}\right)$, where $o p p t r^{\prime \prime}$ is a pointer to an operation $o p^{\prime \prime} \neq o p$, after $C_{S C}^{\prime}$ and before $C$; this follows from the definition of $p^{\prime}$. Notice that the second $\mathrm{SC}(x \rightarrow A[q]$, opptr $)$ performed by $p$ on line 73 is executed after $C_{S C}^{\prime}$, so it cannot be successful. However, this SC is unsuccessful only if a process $\neq p$ performs a successful SC on $x \rightarrow A[q]$ after $C_{L L 2}$ and before it, thus between $C_{S C}^{\prime}$ and $C$, which is a contradiction.

- $C_{S C^{\prime}}$ occurs after $C_{L L 2}$. Notice that $C_{L L}^{\prime}$ precedes $C_{L L 1}$ and $p$ performs a $\mathrm{SC}(\mathrm{x} . \mathrm{A}[\mathrm{q}], \mathrm{opptr}$ ) (on line 70) between $C_{L L 1}$ and $C_{L L 2}$. If this $\mathrm{SC}$ is successful, then the $\mathrm{SC}(x . A[q])$,opptr') performed by $p^{\prime}$ immediately before $C_{S C^{\prime}}$ cannot be successful, which is a contradiction. Otherwise, another process performs a successful SC on $x . A[q]$ after $C_{L L 1}$ and before $p$ performs the $\operatorname{SC}(x . A[q]$,opptr $)$ on line 70, which also prevents the SC performed by $p^{\prime}$ from being successful, which is a contradiction.

Attempts of distinct operations may access the same varrecs. When an attempt att of op accesses a varrec $x$ for the first time by simulating $\operatorname{READDI}(x)$ or $\operatorname{WRITEDI}\left(x,{ }_{-}\right)$, the operation is first announced to $x$ (on line 39) and then ConfLicts (opptr, $x$ ) is called (on line 40, opptr is a pointer to op) to check whether another attempt $a t t^{\prime}$ of a distinct operation $o p^{\prime}$ is concurrently accessing $x$. If this is the case (line 78), $o p^{\prime}$ is either restarted (on line 87) or helped (on lines 82, 88 or 89). Since when $\operatorname{HeLP}\left(o p^{\prime}\right)$ returns, the status of $o p^{\prime}$ is done (Observation 8), in both cases attempt $a t t^{\prime}$ is no longer active when the call to ConfLICTs(opptr, $x$ ) returns. This is precisely what next Lemma establishes.

Lemma 17. Let att, att' be two attempts by two processes denoted $p$ and $p^{\prime}$, respectively, of two operations op, op $p^{\prime}$ owned by $q, q^{\prime}$, where $q \neq q^{\prime}$, respectively. Let $x$ be a varrec. Denote by opptr and opptr' two pointers to op and op $^{\prime}$ respectively. Suppose that:

- in att, $p$ calls ANNOUnCE(opptr, $x)$ and returns from that call, 
- in att ${ }^{\prime}, p^{\prime}$ calls Conflicts $\left(o p p t r^{\prime}, x\right)$ (on line 40) and returns from that call; denote by $C_{D}^{\prime}$ the configuration that follows the termination of CONFLICTS $\left(o p p t r^{\prime}, x\right)$ by $p^{\prime}$.

- $p^{\prime}$ returns from AnNounce $\left(o p p t r^{\prime}, x\right)$ after $p$ returns from AnNounce $(o p p t r, x)$.

Then, if att ${ }^{\prime}$ is active in $C_{D}^{\prime}$, the following hold:

1. att is not active in $C_{D}^{\prime}$;

2. if att is successful, opptr $\rightarrow$ status $=$ done in $C_{D}^{\prime}$.

Proof. Let $C_{A}$ denote the configuration immediately after $p$ returns from Announce $(o p p t r, x)$. Similarly, denote by $C_{A}^{\prime}$ the configuration immediately after $p^{\prime}$ returns from $\operatorname{AnnouncE}\left(o p p t r^{\prime}, x\right)$. We have that $C_{A}^{\prime}$ occurs after $C_{A}$, and $C_{A}^{\prime}$ occurs before $p^{\prime}$ calls Conflicts $\left(o p p t r^{\prime}, x\right)$.

The proof is by contradiction. Let us assume that $a t t^{\prime}$ is active in $C_{D}^{\prime}$ and either att is active in $C_{D}^{\prime}$ or att is successful and opptr $\rightarrow$ status $\neq$ done in $C_{D}^{\prime}$. Consider the execution by $p^{\prime}$ of the call Conflicts $\left(o p p t r^{\prime}, x\right)$, which ends at configuration $C_{D}^{\prime}$. In particular, as $q^{\prime}=o p^{\prime} \rightarrow$ owner $\neq$ op $\rightarrow$ owner $=q$, process $p^{\prime}$ checks whether an operation owned by $q$ has been announced to the varrec pointed to by $x$ (on line 76). We derive a contradiction by examining the steps taken by process $p^{\prime}$ in the iteration of the for loop in which $x \rightarrow A[q]$ is examined.

Let $C$ be a configuration that follows $C_{A}$ and precedes $C_{D}^{\prime}$ or is equal to $C_{D}^{\prime}$. We show that $x \rightarrow A[q]=$ opptr in $C$. On one hand, att is active in configuration $C_{A}$ and thus opptr $\rightarrow$ status $=$ simulating in this configuration. On the other hand, either att is still active in $C_{D}^{\prime}$, or att is successful, but opptr $\rightarrow$ status $\neq$ done in $C_{D}^{\prime}$. Therefore, by Observation 7, the status of op does not change between $C_{A}$ and $C_{D}^{\prime}$ or is changed to $\left\langle\right.$ modifying, $\left.,_{-},-\right\rangle$. Hence, opptr $\rightarrow$ status $\in\left\{\right.$ simulating, $\left\langle\right.$ modifying, $\left.\left.,_{-},-\right\rangle\right\}$in $C$.

In particular the configuration $C_{R A}^{\prime}$ that immediately precedes the read of $x . A[q]$ by $p^{\prime}$ (LL on line 77) occurs after $C_{A}$ and before $C_{D}^{\prime}$. $C_{R A}^{\prime}$ thus occurs after the call of Announce (opptr, $x$ ) by $p$ returns, and the status of $o p$ is not done in this configuration. Therefore, by applying Lemma 16, we have that $A[q]=o p p t r$ in $C_{R A}^{\prime}$.

As attempt $a t t^{\prime}$ is active in $C_{D}^{\prime}$, it is active when $p^{\prime}$ performs ConfliCts $\left(o p p t r^{\prime}, x\right)$. In particular, each VL on opptr $\rightarrow$ status performed by $p^{\prime}$ (on line 80 or 86 ) in the execution of ConfLICTS $\left(o p p t r^{\prime}, x\right)$ returns true. Therefore, $p^{\prime}$ reads the status of the operation pointed to by oppptr (LL(opptr $\rightarrow$ status) on line 79). In the configuration to which this LL is applied, which occurs between $C_{A}$ and $C_{D}^{\prime}$, the status of $o p$ is either simulating or $\langle$ modifying, ,, $\rangle$ for what above stated.

We consider two cases, according to the value read from opptr $\rightarrow$ status by $p^{\prime}$ :

- The read of opptr $\rightarrow$ status by $p^{\prime}$ returns $\left\langle\right.$ modifying, $,_{-},{ }_{-}$. In that case, $p^{\prime}$ calls HelP(opptr) (line 82). In the configuration $C$ in which $p^{\prime}$ returns from this call, opptr $\rightarrow$ status $=$ done (Observation 8). As $C$ is $C_{D}^{\prime}$ or occurs prior to $C_{D}^{\prime}$, but after $C_{A}$, and the status of op is never changed to done between $C_{A}$ and $C_{D}^{\prime}$, this is a contradiction.

- The read of opptr $\rightarrow$ status by $p^{\prime}$ returns simulating (line 83). We distinguish two sub-cases according to the relative priorities of $o p$ and $o p^{\prime}$ :

$-q^{\prime}<q$, i.e., $o p^{\prime}$ has higher priority than $o p$. In this case, $p^{\prime}$ tries to change the status of $o p$ to $\left\langle\right.$ restart,,$\left._{-}\right\rangle$by performing a SC on opptr $\rightarrow$ status with parameter $\left\langle\right.$ restart, opptr $\left.{ }^{\prime}\right\rangle$ (line 87). The $\mathrm{SC}$ is performed in a configuration that follows $C_{A}$ and that precedes $C_{D}^{\prime}$. The SC cannot succeed. Otherwise there is a configuration between $C_{A}$ and $C_{D}^{\prime}$ where opptr $\rightarrow$ status is $\left\langle\right.$ restart, opptr $\left.{ }^{\prime}\right\rangle$. This contradicts the fact that the status of $o p$ is simulating or $\left\langle\right.$ modifying, $\left.,_{-},-\right\rangle$in every configuration between $C_{A}$ and $C_{D}^{\prime}$. Therefore, opptr $\rightarrow$ status has been changed to $\left\langle\right.$ modifying,,,$\left._{-}\right\rangle$ before the SC is performed by $p^{\prime}$. Thus, $p^{\prime}$ calls $\operatorname{HeLP}($ opptr) (on line 88) after performing the unsuccessful SC. When this call returns, opptr $\rightarrow$ status $=$ done (Observation 8 ) which is a contradiction. 
$-q<q^{\prime}$. In that case, $p^{\prime}$ calls HeLP(opptr). As in the previous case, a contradiction can be obtained, since when $p^{\prime}$ returns from this call, opptr $\rightarrow$ status $=$ done (Observation 8), and $p^{\prime}$ returns from the call to $\operatorname{HELP}\left(\right.$ opptr) before $C_{D}^{\prime}$.

In an attempt of op, a new varrec is created each time a CREATEDI() instruction is simulated on line 44. For such a varrec to be later accessed in another attempt, a pointer to it must be either written to the val field of another varrec, or passed as an input parameter to an operation. Moreover, when the varrec is accessed, the status of the operation $o p$ is done.

Lemma 18. Suppose that in att, $p$ creates a varrec $x$. If an instruction $\operatorname{READDI}(x)$ or $\operatorname{WrITEDI}\left(x,{ }_{-}\right)$ is simulated in an attempt att ${ }^{\prime}$ of an operation $o p^{\prime} \neq o p$, then op $\rightarrow$ status $=$ done in the configuration preceding the beginning of the simulation of this instruction.

Proof. Recall that $x$ is allocated to a new shared memory slot (on line 44) and then a dictrec with key a pointer to $x$ is added to the dictionary associated with att (on line 46). While att is active, the dictionary associated with it is private. Hence, in order for a WRITEDI() or READDI() with parameter $x$ to be simulated in $a t t^{\prime}$, the dictionary associated with att has to be made public, which can occur only if att is successful. Moreover, there is a varrec $x^{\prime}$ created by att such that $x^{\prime}$ is written to a varrec that is not created by att, or it is returned by op. This is so, since otherwise, no varrecs created in att can be accessed in any attemp other than att, which contradicts the fact that $x$ is accessed by $a t t^{\prime}$. In the second case, the code (lines 23 and 26) and Observation 8 imply that opptr $\rightarrow$ status $=$ done before a pointer to $x$ is passed as a parameter to $o p^{\prime}$, that is before $a t t^{\prime}$ simulates an access on $x$; so, the claim holds. We continue with the first case. Denote by $W$ the set of varrecs that are written by att but have not been created by it.

In $a t t^{\prime}$, an instruction $\operatorname{WriteDI}\left(x,{ }_{-}\right)$or $\operatorname{READDI}(x)$ is simulated. Since $x$ is a dynamic varrec, this instruction is preceded by a simulation of a READDI() instruction on some data item not created by $a t t^{\prime}$ that returns a pointer to $x$. Assume that the first such instruction $R$ has parameter $y$. We argue that $R$ is the first access of $y$ by $a t t^{\prime}$. This is so since a copy of $y$ is inserted into the dictionary of $a t t^{\prime}$ the first time it is accessed by $a t t^{\prime}$ and any subsequent access of $y$ by $a t t^{\prime}$ returns the value written in the dictionary.

1. $y \in W$. Note that $y$ is neither created in att nor in att ${ }^{\prime}$ but accessed in both attempts. Therefore, Observation 15 implies that the first time it is accessed in att, AnNounce (opptr, y) and Conflicts (opptr, y) are called (lines 39-40). Both calls terminate, as att is successful. Denote by $C_{A}$ and $C_{D}$ the configurations that follow the termination of $\operatorname{AnNOUNCE}(o p p t r, y)$ and Conflicts $(o p p t r, y)$, respectively. Notice that att is active in $C_{D}$. This is due to the fact that att remains active until the SC on line 52 that changes the status of op to $\langle$ modifying, , , $\rangle$ is applied.

Similarly, Observation 15 implies that Announce $\left(o p p t r^{\prime}, y\right)$ and Conflicts $\left(o p p t r^{\prime}, y\right)$ are called when $a t t^{\prime}$ simulates $\operatorname{READDI}(y)$. Both calls terminate, since the simulation of READDI(y) by $a t t^{\prime}$ returns a value. Denote by $C_{A}^{\prime}$ and $C_{D}^{\prime}$ the configurations that follow the termination of Announce $\left(o p p t r^{\prime}, y\right)$ and Conflicts $\left(o p p p t r^{\prime}, y\right)$, respectively. Note that $a t t^{\prime}$ is active in $C_{D}^{\prime}$ since another instruction, namely, $\operatorname{ReADDI}(x)$ or $\mathrm{WRITEDI}\left(x,{ }_{-}\right)$, is simulated later, and the status of $o p^{\prime}$ is validated before a new instruction is simulated (line 49).

If $C_{A}$ occurs before $C_{A}^{\prime}$, it follows from Lemma 17 that opptr $\rightarrow$ status $=$ done in $C_{D}^{\prime}$. Therefore, by Observation 7 , the status of $o p$ is done when the simulation of $\operatorname{READDI}(x)$ or $\operatorname{WriteDI}\left(x,{ }_{-}\right)$starts in att ${ }^{\prime}$. Otherwise, $C_{A}^{\prime}$ occurs before $C_{A}$. In that case, it follows from Lemma 17 that att ${ }^{\prime}$ is not active in $C_{D}$. Since the SC on line 52 by att is executed after $C_{D}$ and $x$ becomes visible to other attempts only after this SC, it is not possible for $a t t^{\prime}$ to access $x$, which is a contradiction.

2. $y \notin W$. In this case, a pointer $p t r_{x}$ to $x$ is written to $y$.val before $y$.val is read in $a t t^{\prime}$. This means that in an attempt $a t t^{\prime \prime} \notin\left\{a t t, a t t^{\prime}\right\}$, an instruction WRITEDI $\left(y, p t r_{x}\right)$ is simulated. Moreover, as in $a t t^{\prime}$, this instruction is preceded by the simulation of a READDI() instruction that returns $x$. We apply inductively the same reasoning to $a t t^{\prime \prime}$ to prove the Lemma. In each induction step, the number of configurations between the creation of $x$ (in att) and the first time a READDI() that returns $x$ is simulated in the attempt considered strictly decreases. This ensures the termination of the induction process. 
Next lemma establishes that in every configuration, no two operations that are in their modifying phase reference the same varrec. This lemma plays a central role in the definition of the state of the data structure at the end of a prefix of the (concurrent) execution.

Lemma 19. Let op,op' denote two distinct operations, and let $C$ be a configuration. Suppose that in $C$, $o p \rightarrow$ status $=\left\langle\right.$ modifying, chgs, $\left.{ }_{-}\right\rangle$and $o p^{\prime} \rightarrow$ status $=\left\langle\right.$ modifying, chgs $\left.{ }^{\prime},{ }_{-}\right\rangle$, where chgs and chgs ${ }^{\prime}$ are pointers to dictionaries $d$ and $d^{\prime}$ respectively. Then there is no dictrec with the same key in both $d$ and $d^{\prime}$.

Proof. Assume, by contradiction, that dictionaries $d$ and $d^{\prime}$ have a dictrec whose key field points to the same varrec $x$ in configuration $C$. Since every process owns at most one operation with status $\neq$ done in every configuration (Observation 9 ), op $\rightarrow$ owner $\neq$ op $\rightarrow$ owner.

Consider a process that changes the status of op to $\left\langle\right.$ modifying, chgs, $\left.{ }_{-}\right\rangle$. This occurs when this process performs a SC on op $\rightarrow$ status (on line 52). Since once the status of an operation is $\left\langle\text { modifying, },_{-},\right\rangle_{-}$, , it can only change to done (Observation 7), and for this SC to be successful, the status of op must be simulating in the configuration in which it is applied, there is a unique such process. Denote by $p$ this process. Before changing the status of $o p$ to 〈modifying, chgs, $\left.{ }_{-}\right\rangle, p$ performs a (successful) attempt of op (lines 36 - 50). Denote att this attempt. Note that the dictionary associated with att is $d$. Hence, a dictrec $\left\langle x,_{-}\right\rangle$is added to $d$ during att. Define similarly attempt att by process $p^{\prime}$, the successful attempt of $o p^{\prime}$ that ends with the SC that changes the status of $o p^{\prime}$ to $\left\langle\right.$ modifying, chgs $\left.{ }^{\prime},{ }_{-}\right\rangle$. As in att, a dictrec $\left\langle x,{ }_{-}\right\rangle$is added to $d^{\prime}$ in $a t t^{\prime}$.

We consider two cases, according to the instructions simulated when a dictrec with a pointer $p t r_{x}$ to $x$ is added in att or $a t t^{\prime}$.

- In both att and $a t t^{\prime}$, some dictrec with key $x$ is added to $d$ when a $\operatorname{READDI}(x)$ or $\operatorname{WriteDI}\left(x,{ }_{-}\right)$ is simulated. By the code, $p$ calls in att $\operatorname{Announce}(o p p t r, x)$ and Conflicts $(o p p t r, x)$ (on lines 39 and 40 , respectively) before adding a dictrec $\left\langle p t r_{x},{ }_{-}\right\rangle$, to its dictionary (on line 42), where opptr is pointing to op. Similarly, $p^{\prime}$ calls in $a t t^{\prime} \operatorname{Announce}\left(o p p t r^{\prime}, x\right)$ and Conflicts $\left(o p p t r^{\prime}, x\right)$, where $o p p t r^{\prime}$ is a pointer to $o p^{\prime}$, and $p^{\prime}$ returns from both calls. Assume without loss of generality that $p^{\prime}$ returns from Announce $\left(o p p t r^{\prime}, x\right)$ after $p$ returns from Announce $(o p p t r, x)$ by $p$. Denote by $C_{D}^{\prime}$ the configuration immediately after $p^{\prime}$ returns from $\operatorname{ConfLICTS}\left(o p p t r^{\prime}, x\right)$. As att is a successful attempt, whose end occurs when $p^{\prime}$ changes the status of $o p^{\prime}$ to $\left\langle\right.$ modifying,,$\left._{-}\right\rangle$, att $t^{\prime}$ is active in $C_{D}^{\prime}$.

Therefore, by Lemma 17, att is not active in $C_{D}^{\prime}$ and, since att is a successful attempt, the status of op is done in this configuration. This contradicts the fact that the status op and $o p^{\prime}$ is $\left\langle\right.$ modifying,,$\left.{ }_{-}\right\rangle$ at $C$ that folows $C_{D}^{\prime}$.

- A dictrec with key $x$ is added to $d$ or $d^{\prime}$ when a CREATEDI() is simulated. Whenever a new varrec is created (on line 44), a distinct shared memory slot is allocated to this varrec. A dictrec record $\left\langle p t r_{x},{ }_{-}\right\rangle$cannot thus be added in both $d$ and $d^{\prime}$ at line 46 when a CREATEDI() instruction is simulated.

Suppose without loss of generality that, in att, $\left\langle x,,_{-}\right\rangle$is added to $d$ on line 46 , as a result of the simulation of a CreateDi() instruction. $p t r_{x}$ is thus added to $d^{\prime}$ the first time a $\operatorname{ReAdDI}(x)$ or $\operatorname{WriteDI}\left(x,{ }_{-}\right)$ instruction for $o p^{\prime}$ is simulated by $p^{\prime}$ in $a t t^{\prime}$. By Lemma 18, op status is done in the configuration immediately before the simulation of this instruction begins. Therefore there is no configuration in which the status of $o p$ and $o p^{\prime}$ is $\left\langle\right.$ modifying,,,$\left._{-}\right\rangle$: a contradiction.

Suppose that att is a successful attempt of $o p$. Hence, the status of $o p$ is changed just after att to $\left\langle\right.$ modifying, chgs, $\left.{ }_{-}\right\rangle$. The changes resulting from the instructions simulated in att are stored in the dictionary pointed to by chgs. While the status of $o p$ is $\left\langle\right.$ modifying, chgs, $\left.{ }_{-}\right\rangle$, some processes try to apply these changes by modifying the value of the varrecs referenced by op (on lines 54-64). Next lemma establishes that the changes described by the dictionary pointed to by chgs are successfully applied by the time that the status of $o p$ is changed to done.

Lemma 20. Suppose that $C_{M}$ is the last configuration in which the status of op is $\left\langle\right.$ modifying,chgs, $\left.{ }_{-}\right\rangle$, where chgs is a pointer to a dictionary $d$ of dictrecs. Let $C$ be a configuration that follows $C_{M}$. For every dictrec $\left\langle p t r_{x}, v\right\rangle$ in $d$, where $p t r_{x}$ is a pointer to $a$ varrec $x, p t r_{x} \rightarrow v a l=v$ in $C$ or there exists a configuration $C^{\prime}$ following $C_{M}$ and preceding $C$ and an operation $o p^{\prime}$ such that op ${ }^{\prime}$ is referencing $x$ in $C^{\prime}$. 
Proof. Let $p$ be the process that successfully performs $\mathrm{SC}\left(\right.$ op $\rightarrow$ status, done) on line 63 just after $C_{M}$. Suppose that in every configuration $C^{\prime}$ following $C_{M}$ and preceding $C$, no operation references $x$. Assume, by contradiction, that $p t r_{x} \rightarrow v a l=v^{\prime} \neq v$ in $C$.

Consider the steps performed by $p$ in the execution of the iteration of the for loop (lines $57-62$ ) that corresponds to the dictrec $\left\langle p t r_{x}, v\right\rangle$. Notice that these steps precede $C_{M}$. In this iteration, $p$ tries to change the val of $x$ to $v$. Since $p$ is the process that changes the status of op to done, it follows that $p$ does not return on lines 58 and 61 . Thus, $p$ executes two $\mathbf{S C}$ instructions $\mathbf{S C}_{1}$ and $\mathbf{S C}_{2}$ on lines 59 and 59 , respectively; let $\mathrm{LL}_{1}$ and $\mathrm{LL}_{2}$ be the matching $\mathrm{LL}$ instructions to these SC. Notice that, for each $i \in\{1,2\}$, there is a successful SC between $\mathrm{LL}_{i}$ and $\mathbf{S C}_{i}$. Let $\mathbf{S C}_{i}^{\prime}$ be this successful $\mathbf{S C}$ (notice that $\mathbf{S C}_{i}^{\prime}$ may be $\mathbf{S C}_{i}$ if $\mathbf{S C}_{i}$ is successful).

Since $p t r_{x} \rightarrow v a l=v^{\prime} \neq v$ in configuration $C$, some process changes $p t r_{x} \rightarrow v a l$ to $v^{\prime}$. Let $p^{\prime}$ be the last process that changes $p t r_{x} \rightarrow$ val to $v^{\prime}$ prior to $C$. By the code, $p^{\prime}$ performs successfully $\operatorname{SC}\left(p t r_{x} \rightarrow v a l, v^{\prime}\right)$ on line 59 or 62 ; denote by $\mathrm{SC}^{\prime}$ this $\mathrm{SC}$ and let $\mathrm{LL}^{\prime}$ and $\mathrm{VL}^{\prime}$ be its mathing LL and VL (which are executed on lines 57 and 58 or 60 and 61 ), respectively. Since $p t r_{x} \rightarrow v a l=v^{\prime} \neq v$ in $C$, either $\mathbf{S C}^{\prime}=\mathbf{S C}_{2}^{\prime}$ or $\mathbf{S C}^{\prime}$ occurs after $S C_{2}^{\prime}$.

The status of $o p^{\prime}$ when $V L^{\prime}$ is executed is $\left\langle\right.$ modifying, $\left.{ }^{\prime} g s^{\prime},{ }_{-}\right\rangle$, where chgs $^{\prime}$ is a pointer to a dictionary that includes a dictrec $\left\langle x, v^{\prime}\right\rangle$, thus $o p^{\prime}$ references $x$ when $V L^{\prime}$ is executed. Since we have assumed that no operation references $x$ in any configuration between $C_{M}$ and $C, V L^{\prime}$ precedes $C_{M}$. By Lemma $19, x$ cannot be referenced by two operations at the same time. Hence, $V L^{\prime}$ occurs before the status of $o p$ is changed to $\left\langle\right.$ modifying, chgs, $\left.{ }_{-}\right\rangle$. In particular, $V L^{\prime}$, and therefore also $\mathrm{LL}^{\prime}$ precedes $\mathrm{LL}_{1}$. Since $S C^{\prime}$ is realized at $S C_{2}^{\prime}$ or after it, $\mathrm{SC}_{1}^{\prime}$ occurs between $\mathrm{LL}^{\prime}$ and $\mathbf{S C}^{\prime}$. Thus, $\mathrm{SC}^{\prime}$ is not successful. This is a contradiction.

Recall that the state of a sequential data structure is a collection of pairs $(x, v)$ where $x$ is a data item and $v$ is a value for that data item. The state of the data structure we consider does not depend on where its data items are stored, so by the value of a pointer we mean which object it points to and not the location of that object in shared memory. The initial state of a sequential data structure consists of its static data items and their initial values.

Initially, there is one varrec for each static data item of the data structure. Each varrec that is created (on line 44) becomes a public dynamic data item if the attempt that creates it is successful. The current value of a varrec in a configuration is the value of its val field, unless the varrec is referenced by an operation $o p$, in which case it is the newval field in dictrec, the dictionary contained in op's status, whose key points to this varrec. Note that, by Lemma 19, in each configuration, each varrec is referenced by at most one operation.

Recall that a varrec is public in configuration $C$ if it corresponds to a varrec of a static data item or there exists a configuration $C^{\prime}$ equal to $C$ or preceding it in which it is referenced by an operation. For every configuration $C$ in $\alpha$, denote by $D_{C}$ the set of pairs $(x, v)$, where $x$ is a public varrec and $v$ is its current value in $C$. Notice that $D_{0}=S_{0}$, where $S_{0}$ is the initial state of the data structure. We establish in Theorem 24 that, after having assign linearization points to operations, $D_{C}$ is the state of the data structure that results if the operations linearized before $C$ are applied sequentially, in order, starting from the initial state, i.e., that $D_{C}=S_{C}$.

If an attempt by $p$ of an operation $o p$ is active in configuration $C$, we define the local state of the data structure in $C$ for the operation and the process that performs the attempt as follows.

Definition 21. For every configuration $C$ and every operation op, if an attempt att by $p$ of op is active in $C$, the local state $L S(C, p, o p)$ of the data structure in configuration $C$ for att is the set of pairs $(x, v)$ such that, in configuration $C$ :

- the dictionary associated with att contains a dictrec $\langle x, v\rangle$ or,

- the dictionary associated with att does not contain any dictrec with key $x$ and $(x, v) \in D_{C}$.

The goal is to capture the state of the data structure after the instructions simulated so far in att are applied sequentially to $D_{C}$. We will indeed establish in Theorem 24 that $L S(C, p, o p)$ is the state of the data 
structure, resulting from the sequential application of the instructions of att simulated thus far by $p$ to $S_{C}$. Operations are linearized as follows:

Definition 22. Each operation is linearized at the first configuration in the execution at which its status is $\langle$ modifying, ,,,$\rangle$.

By the code and the way the linearization points are assigned, it follows that:

Lemma 23. The linearization point of each operation is within its execution interval.

We continue with our main theorem which proves consistency.

Theorem 24 (Linearizability). Let $C$ be any configuration in execution $\alpha$. Then, the following hold:

1. $D_{C}=S_{C}$.

2. Let att be an attempt of an operation op by a process $p$ that is active in $C$ and let $\tau$ be the sequence of instructions of op that have been simulated by $p$ until $C$. Denote by $\rho$ the sequence of the first $|\tau|$ instructions in a sequential execution of op starting from state $S_{C}$. Then, $\rho=\tau$ and $L S(C, p, o p)=$ $S_{C} \tau$, where $S_{C} \tau$ is the state of the data structure if the instructions in $\tau$ are applied sequentially starting from $S_{C}$.

The proof of Theorem 24 relies on the following lemma.

Lemma 25. Let att denote an attempt by $p$ of some operation op. Suppose that in att, $x \rightarrow$ val is read by $p$ while an instruction $\operatorname{READDI}(x)$ is simulated (line 41), let $r$ be this read of $x \rightarrow$ val, let $v$ be the value returned by $r$, and denote by $C_{r}$ the configuration immediately before this read. Then, in every configuration $C$ such that $C$ is $C_{r}$ or some configuration that follows $C_{r}$ and att is active at $C, v$ is the value of $x$ in $D_{C}$.

Proof. Assume, by contradiction, that in some configuration $C_{b}$ between $C_{r}$ and $C$, the value of $x$ in $S_{C_{b}}$ is not $v$. Denote by $C^{\prime}$ the first such configuration, and let $v^{\prime}$ be the value of $x$ in $S_{C^{\prime}}$. Note that $C^{\prime}$ may be configuration $C_{r}$.

By definition of $S_{C^{\prime}}, v^{\prime}$ is the current value of $x$ in $S_{C^{\prime}}$ if either there exists an operation $o p^{\prime}$ whose status is $\left\langle\right.$ modifying, chgs $\left.^{\prime},{ }_{-}\right\rangle$where chgs $^{\prime}$ is pointing to a dictionary that contains a dictrec with key $x$ or no such operation exists and $v^{\prime}=x \rightarrow$ val.

In configuration $C_{r}$, which is equal to $C^{\prime}$ or precedes $C^{\prime}, x \rightarrow v a l=v \neq v^{\prime}$. Since in every configuration $C^{\prime \prime}$ between $C_{r}$ and $C^{\prime}$ (if any), the value of $x$ is $v$ in $S_{C^{\prime \prime}}$, there exists an operation $o p^{\prime}$ whose status is $\left\langle\right.$ modifying, chgs $\left.{ }^{\prime},{ }_{-}\right\rangle$where chgs $^{\prime}$ is pointing to a dictionary that contains a dictrec with key $x$. By Lemma $19, o p^{\prime}$ is unique.

Let $p^{\prime}$ be the process that changes the status of $o p^{\prime}$ from simulating to $\left\langle\right.$ modifying, $\left.c h g s^{\prime},{ }_{-}\right\rangle$. Notice that this occurs before $C^{\prime}$. By the code, it follows that $p^{\prime}$ calls Announce $\left(o p p t r^{\prime}, x\right)$ and Conflicts $\left(o p p t r^{\prime}, x\right)$ where opptr' is pointing to $o p^{\prime}$. Denote by $C_{A}^{\prime}$ and $C_{D}^{\prime}$ the configurations in which $p^{\prime}$ returns from ANNounce $\left(o p p t r^{\prime}, x\right)$ and Conflicts $\left(o p p t r^{\prime}, x\right)$, respectively. Notice that $C_{A}^{\prime}$ and $C_{D}^{\prime}$ precede $C^{\prime}$.

By the code it follows that before reading $x \rightarrow v a l, p$ calls Announce $(o p p t r, x)$ and ConfliCts $(o p p t r, x)$ where opptr is pointing to op. Denote by $C_{A}$ and $C_{D}$ the configurations in which $p$ returns from ANNOUnCE $(o p p t r, x)$ and Conflicts $(o p p t r, x)$, respectively. Notice that $C_{A}$ and $C_{D}$ precede $C_{R}$ and therefore also $C^{\prime}$.

We consider two cases based on the order in which $C_{A}$ and $C_{A}^{\prime}$ occur.

- $C_{A}^{\prime}$ occurs after $C_{A}$. By Lemma 17 , att is not active in $C_{D}^{\prime}$. This is a contradiction, since att is active in configurations $C_{A}$ and $C$, and $C_{D}^{\prime}$ occurs between $C_{A}^{\prime}$ (which, by assumption, follows $C_{A}$ ) and $C$.

- $C_{A}$ occurs after $C_{A}^{\prime}$. The attempt of $o p^{\prime}$ by $p^{\prime}$ in which it calls Announce $\left(o p p t r^{\prime}, x\right)$ and ConFLICTS $\left(o p p t r^{\prime}, x\right)$ is successful, since $p^{\prime}$ is the process that changes the status of $o p^{\prime}$ to $\left\langle\right.$ modifying, _, $\left.{ }_{-}\right\rangle$. Thus, it follows from Lemma 17 that the status of $o p^{\prime}$ in $C_{D}$ is done, contradicting the fact that $o p^{\prime}$ status is $\langle$ modifying, , , $\rangle$ at $C^{\prime}$ that occurs later. 
We finally prove Theorem 24 .

Proof. The proof is by induction on the sequence of configurations in $\alpha$. The claims are trivially true for the initial configuration $C_{0}$. Suppose that the claims is true for configuration $C$ and every configuration that precedes it. Let $C^{\prime}$ be the configuration that immediately follows $C$ in $\alpha$.

We first prove claim 1 . If no operation has its status changed to $\left\langle\right.$ modifying,,,$\left._{-}\right\rangle$between $C$ and $C^{\prime}$, then $D_{C^{\prime}}=D_{C}=S_{C}$. This follows from the definition of $D_{C}$, Lemma 20, and the induction hypothesis (claim 1). Otherwise, denote by op the operation whose status is changed to $\left\langle\right.$ modifying, chgs,,$\left._{-}\right\rangle$in $C^{\prime}$. The status of $o p$ is changed by a SC performed by some process $p$ on line 52 . This SC ends a (successful) attempt att of $o p$ by $p$. Then, in configuration $C^{\prime}$, the dictionary pointed to by chgs is the dictionary associated with att. Hence, by definition of $D_{C^{\prime}}$ and $L S(C, p, o p), D_{C^{\prime}}=L S(C, p, o p)$. By the inductive hypothesis (claim 2), $L S(C, p, o p)=S_{C} \tau$, where $\tau$ is the sequence of instructions simulated by att until $C$. Notice that the last instruction of $\tau$ is the last instruction of $o p$ and $o p$ is the only operation that is linearized at $C^{\prime}$. Thus, by definition of $S_{C^{\prime}}$, it follows that $S_{C} \tau=S_{C^{\prime}}$. Since $L S(C, p, o p)=S_{C} \tau$, and $D_{C^{\prime}}=L S(C, p, o p)$, it follows that $D_{C^{\prime}}=S_{C^{\prime}}$, as needed by claim 1 .

Since by claim $1, D_{C^{\prime}}=S_{C^{\prime}}$, it follows that for each data item in $S_{C^{\prime}}$ there is a unique varrec in $D_{C^{\prime}}$ that corresponds to this data item and vice versa. So, in the rest of proof, we sometimes abuse notation and use $x$ to refer either to a varrec in $D_{C^{\prime}}$ or to a data item in $S_{C^{\prime}}$.

We now prove claim 2. Let att be an attempt by $p$ of some operation op. If att is not active in $C$ but is active in $C^{\prime}$, the step preceding $C^{\prime}$ is a LL that reads the status of op (on lines $28,33,53$ or 64 ). In that case, no step of $o p$ has been simulated until $C^{\prime}$, so $\rho$ and $\tau$ are empty and by definition, $L S\left(C^{\prime}, p, o p\right)=S_{C^{\prime}}$. So, claim 2 holds trivially in this case.

In the remaining of the proof, we assume that att is active in both $C$ and $C^{\prime}$. Denote by $\tau$ and $\tau^{\prime}$ the sequences of instructions of $o p$ simulated in att until $C$ and $C^{\prime}$, respectively. Let $d_{C}$ and $d_{C^{\prime}}$ be the values of the dictionary $d$ that is associated with attempt att, in configurations $C$ and $C^{\prime}$, respectively.

We argue below that two properties, called P1 and P2 below, which are important ingredients of the proof, are true:

P1 Let $C_{i}$ be either $C$ or a configuration that precedes $C$ in which att is active. Let $\tau_{i}$ be the sequence of instructions that have been simulated in att until $C_{i}$. If $x$ is a varrec such that $\operatorname{ReADDI}(x)$ is the first access of $x$ in $\tau_{i}$ then the value of $x$ is the same in states $S_{C_{i}}$ and $S_{C^{\prime}}$.

To prove $\mathrm{P} 1$, denote by $v$ the value returned by the simulation of the first $\operatorname{READDI}(x)$ in $\tau_{i}$. Notice that this is also the value read on line 41 when $\operatorname{READDI}(x)$ is simulated in att. Also, since READDI $(x)$ has been simulated by $C_{i}$, it follows that this read precedes $C_{i}$. Since att is active in configurations $C_{i}$ and $C^{\prime}$, Lemma 25 implies that $v$ is the value of $x$ in both states $S_{C_{i}}$ and $S_{C^{\prime}}$.

P2 Let $C_{i}$ be either $C$ or a configuration that precedes $C$ in which att is active. Denote by $d_{C_{i}}$ the value of $d$ in $C_{i}$ and by $\tau_{i}$ the sequence of instructions that have been simulated in att until $C_{i}$. A dictrec $\langle x, v\rangle$ is contained in $d_{C_{i}}$ if and only if $x$ has been accessed in $\tau_{i}$ and $v$ is the value of $x$ in $S_{C_{i}} \tau_{i}$.

To prove P2, notice that by the code, a dictrec with key $x$ is added to $d$ if and only if an instruction accessing $x$ is simulated (on lines 42 or 46). By the induction hypothesis for $C_{i}$ (claim 2), $S_{C_{i}} \tau_{i}$ is well defined and $L C\left(C_{i}, p, o p\right)=S_{C_{i}} \tau$. Thus, by the definition of $L C\left(C_{i}, p, o p\right),\langle x, v\rangle$ is contained in $d_{C_{i}}$ if and only if $x$ has been accessed in $\tau_{i}$ and $v$ is the value of $x$ in $S_{C_{i}} \tau_{i}$.

Fix any $x$ that att has accessed for the first time by performing READDI $(x)$. Property P1 implies that $x$ has the same value in $S_{C}$ and $S_{C^{\prime}}$. Since we have assumed that operations are deterministic and the state of the data structure does not depend on where its data items are stored, it follows that the first $|\tau|$ instructions of $o p$ are the same and return the same values, independently of whether they are applied in a sequential execution starting from $S_{C}$ or from $S_{C^{\prime}}$. Since, by the induction hypothesis (claim 2), $\tau$ is the same sequence as that containing the first $|\tau|$ instructions of op executed sequentially starting from state $S_{C}, \tau$ is also the same as the sequence of first $|\tau|$ instructions of op executed sequentially starting from state $S_{C^{\prime}}$. Thus, if $\tau=\tau^{\prime}$, claim 2 follows. 
Assume now that $\tau$ and $\tau^{\prime}$ differ, i.e., $\tau^{\prime}=\tau$. ins. Let $C^{\prime \prime}$ be the configuration immediately before the simulation of ins starts. If the simulation of ins starts on line 36, that is, $\tau$ is the empty sequence and thus $\tau^{\prime}=i n s$ and $i n s$ is the first instruction of op executed. Thus, ins is the first instruction of $o p$ when executed sequentially starting from state $S_{C}^{\prime}$. Otherwise, the simulation of ins starts on line 50 . In $C^{\prime \prime}$, the sequence of instructions of $o p$ that have been simulated is $\tau$. The fact that it is instruction ins that is simulated next depends on the input of $o p$, the value $d_{C^{\prime \prime}}$ of the dictionary $d$ in configuration $C^{\prime \prime}$ and op's program. On the other hand, in a sequential execution, the instruction of $o p$ that follows $\tau$ depends only on the input of $o p$, the value of each data item accessed in $\tau$ after $\tau$ has been applied, and op's program. By property $P 2$ applied to $C^{\prime \prime}, d$ contains in $C^{\prime \prime}$ a dictrec $\langle x, v\rangle$ if and only if $x$ is accessed in $\tau$ and $v$ is the value of $x$ in $S_{C^{\prime \prime}} \tau$. Therefore ins is the instruction of op that follows $\tau$ in any sequential execution in which op is applied to $S_{C^{\prime \prime}}$.

Moreover, in a sequential execution of op starting from state $S_{C^{\prime}}, \tau$ is also the sequence of the first instructions of $o p$. Hence, the same data items are accessed by the first $|\tau|$ instructions of $o p$, regardless of whether op is applied to $S_{C^{\prime \prime}}$ or $S_{C^{\prime}}$. Moreover, by property $P 1$ applied to $C^{\prime \prime}$ and the fact that program of $o p$ is deterministic, each of these data items have the same value in $S_{C^{\prime \prime}} \tau$ and $S_{C^{\prime}} \tau$. Therefore, ins is also the next instruction of $o p$ following $\tau$ in any sequential execution in which op is applied to $S_{C^{\prime}}$. We thus conclude that the first $\left|\tau^{\prime}\right|$ instructions of $o p$ when executed starting from state $S_{C^{\prime}}$ in a sequential execution is $\tau^{\prime}$.

By the code, a dictrec with key $x$ is added to $d$ if and only if an instruction accessing $x$ is simulated (on lines 42 or 46). Hence, in configuration $C^{\prime}$, there is a dictrec with key $x$ in $d$ if and only if $x$ is accessed in $\tau^{\prime}$ when $o p$ is applied to $S_{C^{\prime}}$ in a sequential execution. Therefore, the set of varrecs in $L C\left(C^{\prime}, p, o p\right)$ is the same as the set of data items in the state $S_{C^{\prime}} \tau^{\prime}$. Consider two pairs $(x, v) \in L C\left(C^{\prime}, p, o p\right)$ and $(x, u) \in S_{C^{\prime}} \tau^{\prime}$. To complete the proof that $L C\left(C^{\prime}, p, o p\right)=S_{C^{\prime}} \tau^{\prime}$, we show that $u=v$ :

- There is no dictrec with key $x$ in $d$ in configuration $C^{\prime}$, or equivalently, $x$ is not accessed by any instruction of $\tau^{\prime}$ when $o p$ is applied to $S_{C^{\prime}}$ in a sequential execution. Then the value of $x$ in $L C\left(C^{\prime}, p, o p\right)$ is the value of $x$ in $S_{C^{\prime}}$ which is the value of $x$ in $S_{C^{\prime}} \tau^{\prime}$.

- $\tau^{\prime}=\tau$ or $\tau^{\prime}=\tau \cdot$ ins but $x$ is not accessed by ins. In that case, the value $v$ of $x$ in $L C\left(C^{\prime}, p, o p\right)$ is also the value of $x$ in $L C\left(C^{\prime}, p, o p\right)$. By the induction hypothesis, $v$ is also the value of $x$ in $S_{C} \tau$. Since $\tau=\tau^{\prime}$ or ins is not accessing $x, v$ is also the value of $x$ in $S_{C^{\prime}} \tau^{\prime}$.

- $\tau^{\prime}=\tau \cdot$ ins and $x$ is accessed by ins. If ins is $\operatorname{READDI}(x)$ and $x$ is not accessed in $\tau$, it follows from Lemma 25 and the fact that att is active in $C^{\prime}$ that $v$ is the value of $x$ in $S_{C^{\prime}}$. Thus $v$ is also the value of $x$ in $S_{C^{\prime}} \tau^{\prime}$. If ins is $\operatorname{READDI}(x)$ but $x$ is accessed in $\tau, x$ has the same value in $L S(C, p, o p)$ and in $L S\left(C^{\prime}, p, o p\right)$. Since $x$ has also the same value in $S_{C^{\prime}} \tau^{\prime}$ and $S_{C} \tau$, it follows by the induction hypothesis that $x$ has the same value in $L S\left(C^{\prime}, p, o p\right)$ and $S_{C^{\prime}} \tau^{\prime}$.

Finally, if $i n s=\operatorname{WriteDI}(x, v)$ or ins is a CreateDI () that creates $x, x$ has the same value $(v$ or $n i l$ if $i n s=\operatorname{CrEATEDI}())$ in both $L C\left(p, C^{\prime}, o p\right)$ and $S_{C^{\prime}} \tau^{\prime}$.

\subsection{Wait Freedom}

Consider any sequential data structure and suppose there is a constant $M$ such that every sequential execution of an operation applied to the data structure starting from any (legal) state accesses at most $M$ data items. Then we will prove that, in any (concurrent) execution $\alpha$ of our universal construction, DAP-UC, applied to the data structure, every call of PERFORM by a nonfaulty process eventually returns.

Observation 26. For every oprec, tohelp $\left[p^{\prime}\right]$ is initially nil and is only changed to point to oprecs with owner $p^{\prime}$.

This follows from the fact that tohelp $\left[p^{\prime}\right]$ is initialized to nil when the oprec is created (on line 22) and when it is updated (on line 85), opptr' points to an oprec whose owner is $p^{\prime}$, by Observation 10 (line 77). 
We say that op restarts op in an execution if some process calls ConfliCTs (opptr, $x$ ), where opptr points to op and $x$ points to a varrec, and successfully performs $\mathrm{SC}\left(\right.$ opptr ${ }^{\prime} \rightarrow$ status, $\langle$ restart, opptr $\rangle$ ) (on line 87), where opptr' points to $o p^{\prime}$. Note that, by line 84, this can only happen if the owner of $o p$ has higher priority (i.e. smaller identifier) than the owner of $o p^{\prime}$. Thus, an operation cannot restart another operation that has the same owner. Next, we show that an operation cannot restart more than one operation owned by each other process.

Lemma 27. For any operation op and any process $p$ other than its owner, there is at most one time that op restarts an operation owned by $p$.

Proof. Suppose operation $o p$ has restarted operation $o p^{\prime}$ owned by process $p$. Before any process can change the status of $o p^{\prime}$ from $\langle$ restart, opptr $\rangle$ back to simulating (on line 32), where opptr is a pointer to op, it performs $\operatorname{HeLP}(o p p t r)$ on line 31. When this returns, the status of op is done, by Observation 8 .

Consider any process $q$ performing HeLP (opptr) with opptr pointing to op, after the status of op has been set to done. If, when it performs LL on line $79, q$ sees that $o p^{\prime}$ has status simulating, it will see that the status of $o p$ is done, when it performs line 86. Hence, $q$ will not restart $o p^{\prime}$ on line 87.

Conversely, we show that an operation cannot be restarted more than twice by operations owned by a single process.

Lemma 28. For any operation op ${ }^{\prime}$ and for any process $p$ other than its owner, at most two operations owned by $p$ can restart $o p^{\prime}$.

Proof. Let $S$ be the set containing those operations initiated by $p$ that restart $o p^{\prime}$, which is owned by process $p^{\prime} \neq p$. Let opptr' be a pointer to the oprec record of $o p^{\prime}$. Let $|S|=k$ and assume, by the way of contradiction, that $k>2$. Let $o p_{i} \in S, 1 \leq i \leq k$, be the $i$-th operation that restarts $o p^{\prime}$ when a process $q_{i}$ executing an attempt of $o p_{i}$ successfully executes the SC on line 87 for $o p^{\prime}$; let $o p p t r_{i}$ be a pointer to the oprec record of $o p_{i}$. Before doing so, $q_{i}$ set opptr ${ }_{i} \rightarrow$ tohelp $\left.^{\prime} p^{\prime}\right]=$ opptr' $^{\prime}$ (on line 85) and then checked that the status of $o p_{i}$ was still simulating (on line 86); thus, opptr $r_{i} \rightarrow$ tohelp $\left[p^{\prime}\right]$ is written before the completion of $o p_{i}$.

Lemma 27 implies that $o p_{i}$ will not restart any other operation owned by process $p^{\prime}$. Recall that $p$ does not call PERFORM recursively, either directly or indirectly; so, before $o p_{i+1}$ is initiated by $p, p$ 's call of Perform $\left(o p p t r_{i}\right)$ should respond (on line 26). Before this response, $p$ reads opptr $r_{i} \rightarrow$ tohelp$\left.^{\prime} p^{\prime}\right]$ on line 25. Since, the call of $\operatorname{HeLP}\left(\right.$ opptr $\left._{i}\right)$ by $p$ (on line 23) has responded before this read, Observation 8 implies that this read is performed after the status of $o p_{i}$ changed to done; thus, it is performed after $q_{i}$ set opptr $_{i} \rightarrow$ tohelp $\left[p^{\prime}\right]=$ opptr $^{\prime}$

If in the meantime the value of opptr $r_{i} \rightarrow$ tohelp $\left[p^{\prime}\right]$ has not changed, then $p$ calls HeLP $\left(o p p t r^{\prime}\right)$. By Observation 8, the status of $o p^{\prime}$ is done when this call responds. Thus, any subsequent operation owned by $p$ will see the status of $o p^{\prime}$ is done and will not restart it. So, it should be that in the meantime some process $q_{i}^{\prime}$ set opptr $\rightarrow$ tohelp $\left[p^{\prime}\right]=$ opptr $_{i}^{\prime}$, where opptr ${ }_{i}^{\prime} \neq$ opptr $^{\prime}$, while executing an attempt of opptr $r_{i}$. Observation 26 implies that opptr ${ }_{i}^{\prime}$ points to the oprec record of some operation $o p_{i}^{\prime}$ initiated by $p^{\prime} ; o p_{i}^{\prime}$ should be initiated by $p^{\prime}$ before $o p^{\prime}$, since otherwise Observation 9 implies that the status of $o p^{\prime}$ has changed to done (so, any subsequent operation owned by $p$ will see the status of $o p^{\prime}$ is done and will not restart it). Observation 26 implies that the status of any operation initiated by $p^{\prime}$ before opptr (including opptr ${ }_{i}^{\prime}$ ), changed to done before the initiation of opptr', that is before $q_{i}$ sets opptr $r_{i} \rightarrow$ tohelp $\left.^{\prime} p^{\prime}\right]=$ opptr $^{\prime}$, that is before $p$ reads opptr $r_{i} \rightarrow$ tohelp $\left.^{\prime} p^{\prime}\right]$ (on line 25), that is before $p$ initiates opptr $r_{i+1}$.

Now consider any $j, 1<j \leq k$. Notice that $q_{j}^{\prime}$ reads opptr ${ }_{j}^{\prime}$ on line 77 and before it executes line 85, which sets opptr $r_{j} \rightarrow$ tohelp$\left.^{\prime} p^{\prime}\right]=$ opptr $_{j}^{\prime}$, it reads the status of opptr ${ }_{j}^{\prime}$ (on line 79) and checks whether it is still simulating (on line 83). Since, this read is performed after the initiation of $o_{p p t r}$, it follows that before it the status of opptr ${ }_{j}^{\prime}$ has changed to done. So, the check fails and line 85 is not executed; that is a contradiction.

From Lemmas 27 and 28, we get the following result. 
Corollary 29. An operation can be restarted at most $2 *(n-1)$.

Next, we bound the depth of recursion that can occur.

Lemma 30. Suppose that, while executing $\operatorname{HelP}\left(\right.$ opptr $\left._{i}\right)$, a process calls $\operatorname{HeLP}\left(\right.$ opptr $\left._{i+1}\right)$, for $1 \leq i<k$. Then $k \leq n$.

Proof. Process $p$ may perform recursive calls to $\operatorname{HeLP}\left(o p p t r^{\prime}\right)$ on lines $31,82,88$, and 89 . If $p$ calls HelP $\left(\right.$ opptr $\left.{ }^{\prime}\right)$ recursively on line 82 or 88 , then, by Observation 7 , opptr ${ }^{\prime} \rightarrow$ status is either modifying or done, so, this recursive call will eventually return without itself making recursive calls to HeLP.

By line 77 and Observation 10, when line 84 is performed, opptr ${ }^{\prime} \rightarrow$ owner $=p^{\prime}$. From line 84, if $p$ calls $\operatorname{HeLP}\left(\right.$ opptr $\left.r^{\prime}\right)$ recursively on line 89, then opptr $\rightarrow$ owner $>$ opttr $r^{\prime} \rightarrow$ owner.

If opptr ${ }^{\prime} \rightarrow$ status $=\langle$ restart, opptr $\rangle$, then, from lines 87 and 84 , opptr $\rightarrow$ owner $<$ opttr ${ }^{\prime} \rightarrow$ owner. Hence, if $p$ calls $\operatorname{HeLP}\left(o p p t r^{\prime}\right)$ recursively on line 31 , opptr $\rightarrow$ status $=\left\langle\right.$ restart, opptr $\left.{ }^{\prime}\right\rangle$, so, again, opptr $\rightarrow$ owner $>$ opttr' $\rightarrow$ owner.

Thus, in any recursively nested sequence of calls to HeLP, the process identifiers of the owners of the operations with which HELP is called is strictly decreasing, except for possibly the last call. Therefore $k \leq n$.

Lemma 31. Every call of HeLP(opptr) by a nonfaulty process eventually returns.

Proof. Consider any call of HelP(opptr) by a nonfaulty process $p$ where opptr points to op. Immediately prior to every iteration of the while loop on lines 29-63 during HELP(opptr), process $p$ performs LL(opptr $\rightarrow$ status) on line $28,33,53$, or 64 .

If op has status done at the beginning of an iteration, $\operatorname{HeLP}(o p p t r)$ returns immediately. If opptr has status modifying, no recursive calls to HeLP are performed during the iteration. Then, Observation 15 and Theorem 24 (item 1) imply that the dictrecs in a dictionary have different keys (i.e. point to different varrecs) and correspond to different data items accessed by a sequential execution of $o p$ applied to the data structure (lines 38,42 , and 46). Thus, the total number of dictrecs in a dictionary is bounded above by $M$ and, so, at most $M$ iterations of the for loop on lines 56-62 are performed. Hence HeLP(opptr) eventually returns.

If opptr has status restart, then, during an iteration of the while loop, $p$ performs one recursive call to HelP (on line 31) and, excluding this, performs a constant numbers of steps.

Finally, suppose that opptr has status simulating at the beginning of an iteration. Theorem 24 (item 2 ) implies that $p$ simulates a finite number of instructions while it is executing an active attempt of $o p$. After this attempt becomes inactive, the test on line 49 evaluates to true during this iteration, so $p$ may simulate at most one more instruction during this iteration; so, the number of instructions is finite. For each instruction in its program, $p$ performs one iteration of the while loop on lines 37-50, in which it takes a constant number of steps, excluding calls to Conflicts. Observation 15, Theorem 24 (item 2), and the definition of $M$, imply that ConfLICTS can be called at most $M$ times during an active attempt of op. Then, Theorem 24 (item 2) imply that process $p$ performs a constant number of steps and at most one recursive call to Help (on line 82, 88, or 89) each time it calls Conflicts. Thus, excluding the recursive calls to HeLP, this iteration of the while loop on lines 29-63 eventually completes.

If $p$ does not return on line 65 after exiting from the while loop or on line 58 or 61 , it tries to change opptr $\rightarrow$ status via an SC on line 32, 52, or 63 . Therefore, each time $p$ performs an iteration of the while loop on lines 29-63, opptr $\rightarrow$ status changes. It follows from Observation 7 and Corollary 29 that $p$ performs at most $2 n$ complete iterations of this while loop during HeLP(opptr).

By Lemma 30, the depth of recursion of calls to HeLP is bounded. Therefore, the call of HeLP(opptr) by $p$ eventually returns.

Finally, we prove wait freedom:

Theorem 32. Every call of PERFORM by a nonfaulty process eventually returns. 
Proof. Consider any call of Perform by a nonfaulty process. In PERForm, the process calls Help at most $n$ times (excluding recursive calls), each time for an oprec owned by a different process It follows from Lemma 31 that all these instances of HELP eventually return. Thus, this call of PERFORM eventually returns.

\subsection{Disjoint access parallelism}

As in the other part of the proof, we consider an execution $\alpha$ of our universal construction applied to some data structure. Recall that the execution interval $I_{o p}$ of an operation op starts with the first step of the corresponding call to Perform() and terminates when this call returns. In the following to simplify the presentation we denote Perform $(o p)$ the call to Perform corresponding to operation op.

Let $C_{o p}$ be the configuration immediately after $p$ performs line 22, that is, immediately after an oprec has been initialized for $o p$, and let $C_{o p}^{\prime}$ be the first configuration at which the status of op is $\langle$ modifying, , , $\rangle$. Note that $C_{i^{\prime}}$ is the configuration at which op is linearized, see Definition 22.

Let $\mathcal{S}=\left\{S_{C} \mid C\right.$ is between $C_{o p}$ and $\left.C_{o p}^{\prime}\right\}$. Then, for the data set $D S(o p)$ of $o p$, it holds that $D S(o p)=$ $\cup_{S_{C} \in \mathcal{S}}\left\{\right.$ set of data items accessed by op when executed sequentially starting from $\left.S_{C}\right\}$.

We recall also the definition of the conflict graph of an execution interval $I$. The conflict graph is an undirected graph, where vertices represent operations whose execution interval overlaps $I$ and an edge connects two operations whose data sets intersect. Given two operations op and $o p^{\prime}$, we denote by $C G\left(o p, o p^{\prime}\right)$ the conflict graph of the minimal execution interval that contains $I_{o p}$ and $I_{o p^{\prime}}$. Finally, recall that we say that two processes contend on a base object $b$ if they both apply a primitive on $b$, and at least one of these primitives is non-trivial.

Recall that an attempt of an operation op by a process $p$ is a longest execution interval that begins when $p$ performs LL on op $\rightarrow$ status on line 28,33,53 or 64 that returns simulating and during which op $\rightarrow$ status does not change.

Lemma 33. When Announce $(o p p t r, x)$ is called, the data item $x$ is in the data set of the operation to which opptr points.

Proof. Let $C$ be the configuration before $p$ calls Announce $(o p p t r, x)$ at which $p$ last performs an LL or a successful VL on opptr $\rightarrow$ status (on lines 28, 33, or 49). By the code, such a configuration $C$ exists, and if $p$ performs an LL at $C$, this LL returns simulating. Hence, an attempt att of op by $p$, the operation pointed to by opptr, is active in configuration $C$. It thus follows from Theorem 24(2) that the sequence of instructions $\tau$ of $o p$ that have been simulated before $C$ is the same as in a sequential execution of $o p$ applied to $S_{C}$. Hence, as in the concurrent execution, ANNOUnCE $(o p p t r, x)$ is called in a simulation of a write to or of a read from $x$ following $\tau, x$ is also accessed in the sequential execution of the first instructions $\tau$ of op applied to $S_{C}$. Therefore, $x \in D S(o p)$.

Inspecting the code of ANNOUNCE, we then obtain:

Corollary 34. If $x \rightarrow A[p] \neq n i l$, then the data item $x$ is in the data set of the operation to which $x \rightarrow A[p]$ points.

Observation 35. If a process executes a successful $\mathrm{VL}($ opptr $\rightarrow$ status) while performing Announce $(o p p t r, x)$ or Conflicts $($ opptr, $x)$, then the oprec to which opptr is pointing has status simulating.

This is because a process only calls Announce (opptr, $x$ ) (on line 39) and Conflicts (opptr, $x$ ) (on line 40 ) if opptr $\rightarrow$ status was simulating (line 34) when $p$ last executed LL(opptr $\rightarrow$ status) (on line 28, 33, or $53)$.

When helping an operation $o p$, process $p$ may starts helping another operation $o p^{\prime}$. This occurs for example when a conflict between the two operations is discovered by $p$, that is, when the two operations access the same varrec. Next Lemma shows that indeed, when $p$ calls $\operatorname{HeLP}\left(o p^{\prime}\right)$ while executing $\operatorname{HeLP}(o p)$, the datasets of $o p$ and $o p^{\prime}$ share a common element. 
Suppose that $p$ calls $\operatorname{HeLP}(o p p t r)$ and $\operatorname{HeLP}\left(o p p t r^{\prime}\right)$, where opptr and opptr' are pointers to operations $o p$ and $o p^{\prime}$, respectively. Denote by $I$ the execution interval of Help $(o p p t r)$. We say that HelP $\left(o p p t r^{\prime}\right)$ is directly called by $p$ after $\operatorname{HelP}(o p p t r)$ if $p$ calls HelP $\left(o p p t r r^{\prime}\right)$ in $I$ and every other call to HelP previously made in by $p$ in $I$ has returned when $\operatorname{HeLP}\left(o p p t r^{\prime}\right)$ is called by $p$.

Lemma 36. If HeLP (opptr') with opptr' pointing to op' is called directly by $p$ after calling HeLP(opptr) with opptr pointing to op, then $D S(o p) \cap D S\left(o p^{\prime}\right) \neq \emptyset$.

Proof. In an instance of $\operatorname{HeLP}(o p p t r)$ by $p$, where opptr is pointing to op, $\operatorname{HeLP}\left(o p p t r^{\prime}\right)$ with opptr $r^{\prime}$ pointing to $o p^{\prime}$ may be called on line 31, when $p$ discovers that op has been restarted, or in the resolution of the conflicts for some varrec $x$, when $p$ executes Conflicts (opptr, $x$ ) (lines 82,88 or 89 ). We consider these two cases separately:

- Help $\left(o p p t r^{\prime}\right)$ is called in the execution of Conflicts $(o p p t r, x)$. Before calling Conflicts $(o p p t r, x)$, $p$ calls Announce $(o p p t r, x)$ (line 39). Therefore, it follows from Lemma 33 that $x \in D S(o p)$. For $\operatorname{Help}\left(o p p t r^{\prime}\right)$ to be called in Conflicts $(o p p t r, x)$, opptr' is read from $x \rightarrow A\left[q^{\prime}\right]$, where $q^{\prime}$ is the owner of $o p^{\prime}$ (LL on line 77). Hence, $o p^{\prime}$ has been previously announced to $x$, from which we conclude by corollary 34 that $x \in D S\left(o p^{\prime}\right)$.

- Help $\left(o p p t r^{\prime}\right)$ is called on line 31. This means that some process $p^{\prime}$ has changed the status of op to $\left\langle\right.$ restart, opptr $\left.{ }^{\prime}\right\rangle$ (SC on line 87). $\quad p^{\prime}$ thus calls ConfLICTS $\left(o p p t r^{\prime}, x\right)$ for some varrec $x$ in which it applies a successful $\mathrm{SC}\left(\right.$ opptr, $\left\langle\right.$ restart, opptr $\left.\left.{ }^{\prime}\right\rangle\right)$. By the code of ConfLICTS, this implies that opptr is read from $x \rightarrow A[q]$, where $q$ is the owner of op (LL on line 77). Thus, op has been announced to $x$, from which we have by Corollary 34 that $x \in D S(o p)$. Moreover, $p^{\prime}$ calls Conflicts $\left(o p p t r^{\prime}, x\right)$ after returning from a call to AnNouncE $\left(o p p t r^{\prime}, x\right)$. Hence, by Lemma 33, $x \in D S\left(o p^{\prime}\right)$.

When a process $p$ is performing an operation $o p$, i.e., $p$ has called PeRform $(o p)$ but has not yet returned from that call, it may access oprecs of operations $o p^{\prime} \neq o p$. We show that if $p$ applies a non-trivial primitive to an oprec $o p^{\prime} \neq o p$ then the execution interval $I_{o p^{\prime}}$ of that operation overlaps the execution interval $I_{o p}$ of op.

Lemma 37. If $p$ applies a non-trivial primitive to an oprec op ${ }^{\prime}$ in $I_{o p}, I_{o p^{\prime}} \cap I_{o p} \neq \emptyset$.

Proof. A non-trivial primitive may be applied to oprec $o p^{\prime}$ on line 32, 52, 55, 63 in the code of HeLP or on lines 85 or 87 in the code of Conflicts. The non-trivial primitive applied by $p$ on line 32,52 or 63 is a SC that aims at changing the status of $o p^{\prime}$ to simulating, $\left\langle\right.$ modifying,,$\left._{-},\right\rangle$or done respectively. On line 55 , the output of $o p^{\prime}$ is changed. Any of these steps, if applied by $p$, is preceded by an LL(opptr ${ }^{\prime} \rightarrow$ status) by $p$ (on lines $28,33,53$ or 64 ), where $o p p t r^{\prime}$ is pointing to $o p^{\prime}$. The value returns by this LL is $\neq d o n e$. Therefore, in the configuration at which this LL is applied, the call of $\operatorname{PERFORM}\left(o p^{\prime}\right)$ has not yet returned. Hence, $I_{o p} \cap I_{o p^{\prime}} \neq \emptyset$.

In the remaining case, $p$ writes opptr ${ }^{\prime}$ to opptr $\rightarrow$ tohelp $\left[p^{\prime}\right]$ on line 85 or applies $\mathrm{SC}\left(\right.$ opptr $^{\prime} \rightarrow,\left\langle\right.$ restart,$\left.\left.{ }_{-}\right\rangle\right)$ on line 87 . Here also, before these steps, an LL(opptr' $\rightarrow$ status) by $p$ occurs (on line 79) and this LL returns a value $\neq$ done. As above, we then conclude that $I_{o p} \cap I_{o p^{\prime}} \neq \emptyset$.

Lemma 38. If $p$ applies a primitive to a varrec $x$ in $I_{o p}$, there exists an operation op' such that $x \in D S\left(o p^{\prime}\right)$, $I_{o p^{\prime}} \cap I_{o p} \neq \emptyset$ and $p$ calls $\operatorname{HeLP}\left(o p p t r^{\prime}\right)$ where opptr' is pointing to op ${ }^{\prime}$.

Proof. Let $x$ denote a varrec accessed by $p$. By the code, $x$ is accessed in one of the following cases:

- The step in which $p$ accesses $x$ occurs in a call to Announce $\left(o p p t r^{\prime}, x\right)$ (lines $68,70,71$, or 73 ), in a call to Conflicts $\left(o p p t r^{\prime}, x\right)$ (line 77) where opptr' is pointing to some operation $o p^{\prime}$, or in the simulation of $\operatorname{READDI}(x)$ on behalf of $o p^{\prime}$ (line 41). Each of these accesses to $x$ occurs after $p$ has called Announce $\left(o p p t r^{\prime}, x\right)$. Therefore, by Lemma 33, $x \in D S\left(o p^{\prime}\right)$. Moreover, before applying any of these steps, $p$ has verified that the status $o p^{\prime}$ is $\neq$ done (by applying a LL on opptr ${ }^{\prime} \rightarrow$ status on line 28,33 or 53). More precisely, consider the last configuration $C$ at which $p$ applies LL(opptr $\rightarrow$ status) 
before accessing $x$. Such a step occurs since the first step following a call to HELP $\left(o p p t r^{\prime}\right)$ is a LL on opptr' $\rightarrow$ status (line 28). This last LL must returns simulating since $p$ has to pass the test on line 34 before applying any step considered in the present case. Therefore, in $C$, the call to Perform $\left(o p^{\prime}\right)$ has not returned, from which we have $I_{o p} \cap I_{o p^{\prime}} \neq \emptyset$.

- The step in which $p$ accesses $x$ is a LL, VL or SC on the val field of $x$ (lines $57,58,59,60,61$ or 62 ). Before applying any of these steps, $p$ performs a $\operatorname{LL}\left(\right.$ opptr $^{\prime} \rightarrow$ status) (on lines 28, 33 or 53), where $o p p t r^{\prime}$ is pointing to $o p^{\prime}$, which returns $\left\langle\right.$ modifying, chgs $\left.{ }^{\prime}{ }_{-}\right\rangle$since the test on line 54 is passed. In the configuration in which this LL is applied, the calls to $\operatorname{Perform}(o p)$ and $\operatorname{PeRForm}\left(o p^{\prime}\right)$ have not returned, hence $I_{o p} \cap I_{o p^{\prime}} \neq \emptyset$.

Consider the dictionary $d^{\prime}$ pointed to by chgs'. Note that $x$ is the key of a dictrec in $d^{\prime}$. Hence, in a successful attempt of $o p^{\prime}$ by some process $p^{\prime}$, a dictrec with key $x$ is added to the dictionary associated with that attempt (on line 42 or 46 ) when an instruction of $o p^{\prime}$ simulated. Therefore, it follows from Theorem 24 that $x \in D S\left(o p^{\prime}\right)$.

Lemma 39. If $p$ calls $\operatorname{HeLP}\left(o p p t r^{\prime}\right)$ in $I_{o p}$, where opptr ${ }^{\prime}$ is pointing to op $p^{\prime}$, then $I_{o p} \cap I_{o p^{\prime}} \neq \emptyset$.

Proof. Process $p$ can only call HeLP $\left(o p p t r{ }^{\prime}\right)$ on line 23, line 25, line 31, line 82, line 88 or line 89 . If $p$ calls $\operatorname{HeLP}\left(o p p t r^{\prime}\right)$ on line 23, $o p^{\prime}=o p$ and the Lemma holds.

If $p$ calls $\operatorname{HeLP}\left(o p p t r^{\prime}\right)$ on line 25, a conflict with $o p^{\prime}$ has been detected by some process $q$ and $q$ has tried to restart $o p^{\prime}$. More precisely, there exists some process $q$, and a varrec $x$ such that $q$ calls Conflicts $($ opptr,$x)$ and, before returning from that call, writes opptr' to opptr $\rightarrow$ tohelp $[p]$ (line 85), where opptr is pointing to op. By the code, before calling Conflicts $(o p p t r, x), q$ verifies that the status of op is simulating by applying a LL on opptr $\rightarrow$ status. Denote by $C_{L L}$ the last configuration that precedes the call to Conflicts (opptr, $x$ ) at which a LL(opptr $\rightarrow$ status $)$ is applied by $q$. opptr $\rightarrow$ status $=$ simulating at $C$. Moreover, it follows from the code of Conflicts that before writing to opptr $\rightarrow$ tohelp $[p]$, $q$ performs a successful VL(opptr $\rightarrow$ status $)$ on line 80 . Let $C_{V L}$ denote the configuration at which this step is applied. By observation 35, opptr $\rightarrow$ status $=$ simulating in $C_{V L}$ and has not changed since $C_{L L}$. In its previous step, $q$ reads opptr $\rightarrow$ status (line 79), and the value it gets back is simulating, since the test on line 83 is later passed. Therefore, there exists a configuration between $C_{L L}$ and $C_{V L}$ in which opptr ${ }^{\prime} \rightarrow$ status $=$ simulating, from which we conclude that $I_{o p} \cap I_{o p^{\prime}} \neq \emptyset$.

$\operatorname{HeLP}\left(o p p t r^{\prime}\right)$ is called on line 31. As in the previous case, a process $q^{\prime}$ performs the successful SC that changes opptr $\rightarrow$ status to $\left\langle\right.$ restart, opptr $\left.{ }^{\prime}\right\rangle$ (on line 87). This occurs when $q^{\prime}$ is executing ConFLICTS $\left(o p p t r^{\prime}, x\right)$ for some varrec $x$. The same reasoning as in the previous case (inverting opptr and opptr' ${ }^{\prime}$ can be used to establish the existence of a configuration in which opptr $\rightarrow$ status $=$ opptr ${ }^{\prime} \rightarrow$ status $=$ simulating, from which it follows that $I_{o p} \cap I_{o p^{\prime}} \neq \emptyset$.

Otherwise, process $p$ calls $\operatorname{HeLP}\left(o p p t r^{\prime}\right)$ on line 82, 88 or 89 . Before calling $\operatorname{HeLP}\left(o p p t r^{\prime}\right)$ on any of these lines, $p$ has read the status of $o p^{\prime}\left(\operatorname{LL}\left(o p p t r^{\prime} \rightarrow\right.\right.$ status $)$ on line 79$)$, and this LL returns a value $\neq$ done (By the tests on line 82 or line 83, opptr $\rightarrow$ status has to be simulating or $\left\langle\right.$ modifying,,$\left._{-},\right\rangle$in order for $p$ to call $\operatorname{HeLP}\left(o p p t r^{\prime}\right)$ on line 82,88 or 89$)$. As this occurs before $p$ returns from the call of PERFORM(op), $I_{o p} \cap I_{o p^{\prime}} \neq \emptyset$.

Lemma 40. Suppose that $p$ applies a primitive operation to an oprec op $p^{\prime}$ after calling HeLP(op) and before returning from that call. Denote by $C$ and $C^{\prime}$ the configuration at which $\operatorname{HeLP}(o p)$ is called and the primitive is applied respectively. If every call by $p$ to $\operatorname{HeLP}()$ that occurs between $C$ and $C^{\prime}$ returns before $C^{\prime}$ then op $=o p^{\prime}$ or $D S(o p) \cap D S\left(o p^{\prime}\right) \neq \emptyset$.

Proof. Suppose that $o p \neq o p^{\prime}$. By the code, $p$ accesses $o p$ while executing ConfliCTs $(o p p p t r, x)$ where $x$ is a varrec and opptr is pointing to op. Since every call to Conflicts $(o p p p t r, x)$ is preceded by a call to Announce $(o p p t r, x)$ (lines 39 and 40), it follows from Lemma 33 that $x \in D S(o p)$. $o p^{\prime}$ is accessed by $p$ via the announce array $x \rightarrow A$. Hence $o p^{\prime}$ has been announced to $x$ and thus by corollary $34, x \in D S\left(o p^{\prime}\right)$. 
Theorem 41. Let $b$ be a base object and let op, op $p^{\prime}$ be two operations. Suppose that $p$ and $p^{\prime}$ apply a primitive on $b$ in $I_{o p}$ and $I_{o p^{\prime}}$ respectively. Then, if at least one of the primitives is non-trivial, there is a path between op and $o p^{\prime}$ in $C G\left(o p, o p^{\prime}\right)$.

Proof. Base object $b$ is a field of either an oprec or a varrec, a dictrec or a statrec. A statrec can only be accessed through the unique oprec that points to it. A dictrec can only be accessed through the unique statrec that points to the unique dictionary that contains it. Thus to access $b, p$ and $p^{\prime}$ have to access the same oprec or the same varrec. We consider these two cases separately:

- $p$ and $p^{\prime}$ access the same oprec $o p^{*}$. Suppose that $o p^{*}$ is accessed by $p$ and $p^{\prime}$ while in some instances of $\operatorname{HeLP}()$. That is, there exists an operation $o p_{1}$ such $p$ calls HelP $\left(o p p t r_{1}\right)$, where opptr $r_{1}$ is pointing to $o p_{1}$, and has not returned from that call when $o p^{*}$ is accessed. Moreover, when it accesses $o p^{*}, p$ has returned from each of its calls to HELP that are initiated after the call to HeLP $\left(o p p t r_{1}\right)$ and before the access of $o p^{*}$.

This also holds for $p^{\prime}$ for some operation $o p_{1}^{\prime}$. Thus, there exists two chains of operations $\langle o p=$ $\left.o p_{k}, \ldots, o p_{1}\right\rangle$ and $\left\langle o p=o p_{k^{\prime}}^{\prime}, \ldots, o p_{1}^{\prime}\right\rangle$ such that:

- $\forall i, 1 \leq i \leq k, \forall i^{\prime}, 1 \leq i^{\prime} \leq k^{\prime}: p$ calls $\operatorname{HeLP}\left(\right.$ opptr $\left._{i}\right)$ and $p^{\prime}$ calls $\operatorname{HeLP}\left(\right.$ opptr $\left._{i^{\prime}}^{\prime}\right)$ where opptr $r_{i}$ and $o p p t r_{i^{\prime}}^{\prime}$ are pointing to $o p_{i}$ and $o p_{i^{\prime}}^{\prime}$ respectively;

- $\forall i, 2 \leq i \leq k, \forall i^{\prime}, 2 \leq i^{\prime} \leq k^{\prime}$ : after calling $\operatorname{HeLP}\left(o p p t r_{i}\right)$, and before returning from this call, $p$ calls directly $\operatorname{HeLP}\left(o p p t r_{i-1}\right)$. Similarly, after calling $\operatorname{HeLP}\left(o p p t r_{i^{\prime}}^{\prime}\right)$, and before returning from this call, $p^{\prime}$ calls directly $\operatorname{HeLP}\left(o p p t r_{i^{\prime}-1}^{\prime}\right)$.

It thus follows from the second property that for each $i, 2 \leq i \leq k, \operatorname{HELP}\left(\right.$ opptr $\left._{i-1}\right)$ is called directly in an attempt of $o p_{i}$, from which we derive by Lemma 36 that $D S\left(o p_{i}\right) \cap D S\left(o p_{i-1}\right) \neq \emptyset$. Moreover, it follows from Lemma 39 that $I_{o p} \cap I_{o p_{i}} \neq \emptyset$, for each $i, 1 \leq i \leq k$. Therefore, operations $o p=$ $o p_{k}, \ldots, o p_{1}$ are vertexes of the graph $C G\left(o p, o p^{\prime}\right)$ and there is path from $o p=o p_{k}$ to $o p_{1}$. Similarly, $o p=o p_{k^{\prime}}^{\prime}, \ldots, o p_{1}^{\prime}$ are vertexes of the graph $C G\left(o p, o p^{\prime}\right)$ and there is path from $o p^{\prime}=o p_{k^{\prime}}^{\prime}$ to $o p_{1}^{\prime}$.

$o p^{*}$ is also a vertex of $G C\left(o p, o p^{\prime}\right)$ because, as $p$ or $p^{\prime}$ applies a non-trivial primitive to $o p^{*}, I_{o p} \cap I_{o p^{*}} \neq \emptyset$ or $I_{o p^{\prime}} \cap I_{o p^{*}} \neq \emptyset$ by Lemma 37. $p$ applies a primitive to $o p^{*}$ after calling $\operatorname{HeLP}\left(o p p t r_{1}\right)$ and before returning from this call. Moreover, when this step is applied, every call to $\operatorname{HeLP}()$ by $p$ that follows the call of $\operatorname{HeLP}\left(o p p t r_{1}\right)$ has returned. Hence by Lemma 40, op $p_{1}=o p^{*}$ or $D S\left(o p_{1}\right) \cap D S\left(o p^{*}\right) \neq \emptyset$. Similarly, $o p_{1}^{\prime}=o p^{*}$ or $D S\left(o p_{1}^{\prime}\right) \cap D S\left(o p^{*}\right) \neq \emptyset$. We conclude that there is a path between $o p$ and $o p^{\prime}$ in $G C\left(o p, o p^{\prime}\right)$.

If $o p^{*}=o p$ or $o p^{*}=o p^{\prime}$, one chain consists in a single operation, namely $o p^{*}$. The reasoning above is still valid.

Finally, $o p^{*}$ may be accessed by $p$ or $p^{\prime}$ on line 25 , when $p$ or $p^{\prime}$ helps an operation that may have been restarted by some process helping $o p$ or $o p^{\prime}$ respectively. Without loss of generality, assume that $o p^{*}$ is accessed in this way, that is $p^{\prime}$ accesses $o p^{*}$ by reading tohelp $\left[p^{*}\right]$, where $p^{*}$ is the owner of $o p^{*}$. As $p$ next calls $\operatorname{HeLP}\left(o p p t r^{*}\right)$, where opptr* is pointing to $o p^{*}$, it follows from Lemma 39 that $I_{o p} \cap I_{o p^{*}} \neq \emptyset$. Therefore, $o p^{*}$ is a vertex of the graph $C G\left(o p, o p^{\prime}\right)$. Consider the step in which $o p p t r^{*}$ is written to opptr $\rightarrow$ tohelp $\left[p^{*}\right]$ (line 85). This occurs while Conflicts $(o p p t r, x)$ is executed, for some varrec $x$. By Lemma 33 and the fact that the call Conflicts $(o p p t r, x)$ is preceded by a call to Announce (opptr, $x), x \in D S(o p)$. Moreover, by the code of $\operatorname{Conflicts}()$, $o p^{*}$ has been announced in to $x$, and thus by corollary $34, x \in D S\left(o p^{*}\right)$. Hence $o p$ and $o p^{*}$ are connected in $C G\left(o p, o p^{\prime}\right)$. Depending on how $o p^{*}$ is accessed by $p^{\prime}$, the same reasoning or the reasoning above can be used to show that there is a path between $o p^{*}$ and $o p^{\prime}$ in $C G\left(o p, o p^{\prime}\right)$. Therefore, there is a path between $o p$ and $o p^{\prime}$ in $C G\left(o p, o p^{\prime}\right)$.

- $p$ and $p^{\prime}$ access the same varrec $x^{*}$. By Lemma 38, there exists $o p_{1}, o p_{1}^{\prime}$ such that (1) $p$ calls HelP $\left(o p_{1}\right)$ and $p^{\prime}$ calls $\operatorname{HeLP}\left(o p_{1}^{\prime}\right),(2) x^{*} \in D S\left(o p_{1}\right) \cap D S\left(o p_{1}^{\prime}\right)$ and (3) $I_{o p} \cap I_{o p_{1}} \neq \emptyset$ and $I_{o p} \cap I_{o p_{1}^{\prime}} \neq \emptyset$. 
If $o p_{1}^{\prime}=o p_{1}=o p^{*}, p$ and $p^{\prime}$ access the same oprec $o p^{*}$. In the proof of the previous item, we use the fact that $p$ or $p^{\prime}$ applies a non-trivial primitive to $o p^{*}$ only to show that $I_{o p^{*}} \cap I_{o p} \neq \emptyset$ or $I_{o p^{*}} \cap I_{o p^{\prime}} \neq \emptyset$. Here, we already known that this holds. Therefore, by the same argument as in the first case, we conclude that there is a path between $o p$ and $o p^{\prime}$ in $C G\left(o p, o p^{\prime}\right)$.

If $o p_{1}^{\prime} \neq o p_{1}$, we consider the two chains of operations chains of operations $\left\langle o p=o p_{k}, \ldots, o p_{1}\right\rangle$ and $\left\langle o p=o p_{k^{\prime}}^{\prime}, \ldots, o p_{1}^{\prime}\right\rangle$ defined as in the first case. By the same reasoning as in the first case, each of these operations is a vertex and $\left(o p_{i}, o p_{i-1}\right),\left(o p_{i^{\prime}}^{\prime}, o p_{i^{\prime}-1}\right)$ are edges of $C G\left(o p, o p^{\prime}\right)$, for each $i, i^{\prime}: 2 \leq$ $i \leq k, 2 \leq i^{\prime} \leq k^{\prime}$. Since $D S\left(o p_{1}\right) \cap D S\left(o p_{1}^{\prime}\right) \neq \emptyset$, we conclude that $o p$ and $o p^{\prime}$ are connected by a path in $C G\left(o p, o p^{\prime}\right)$.

\section{References}

[1] Y. Afek, D. Dauber, and D. Touitou. Wait-free made fast. In Proceedings of the twenty-seventh annual ACM symposium on Theory of computing, STOC '95, pages 538-547, New York, NY, USA, 1995. ACM.

[2] Y. Afek, M. Merritt, G. Taubenfeld, and D. Touitou. Disentangling multi-object operations (extended abstract). In Proceedings of the sixteenth annual ACM symposium on Principles of distributed computing, PODC '97, pages 111-120, New York, NY, USA, 1997. ACM.

[3] J. H. Anderson and M. Moir. Universal constructions for multi-object operations. In Proceedings of the fourteenth annual ACM symposium on Principles of distributed computing, PODC '95, pages 184-193, New York, NY, USA, 1995. ACM.

[4] J. H. Anderson and M. Moir. Universal constructions for large objects. IEEE Transactions on Parallel and Distributed Systems, 10(12):1317-1332, dec 1999.

[5] H. Attiya and E. Dagan. Universal operations: unary versus binary. In Proceedings of the fifteenth annual ACM symposium on Principles of distributed computing, PODC '96, pages 223-232, New York, NY, USA, 1996. ACM.

[6] H. Attiya and E. Hillel. Built-in coloring for highly-concurrent doubly-linked lists. In S. Dolev, editor, Distributed Computing, 20th International Symposium, DISC 2006, Stockholm, Sweden, September 1820, 2006, Proceedings, volume 4167 of Lecture Notes in Computer Science, pages 31-45. Springer, 2006.

[7] H. Attiya and E. Hillel. Highly-concurrent multi-word synchronization. In Proceedings of the 9th international conference on Distributed computing and networking, ICDCN'08, pages 112-123, Berlin, Heidelberg, 2008. Springer-Verlag.

[8] H. Attiya and E. Hillel. Single-version stms can be multi-version permissive. In Proceedings of the 12th international conference on Distributed computing and networking, ICDCN'11, pages 83-94, Berlin, Heidelberg, 2011. Springer-Verlag.

[9] H. Attiya, E. Hillel, and A. Milani. Inherent limitations on disjoint-access parallel implementations of transactional memory. In Proceedings of the twenty-first annual symposium on Parallelism in algorithms and architectures, SPAA '09, pages 69-78, New York, NY, USA, 2009. ACM.

[10] G. Barnes. A method for implementing lock-free shared data structures. In Proceedings of the 5th ACM Symposium on Parallel Algorithms and Architectures, pages 261-270, 1993.

[11] V. Bushkov, R. Guerraoui, and M. Kapalka. On the liveness of transactional memory. In Proceedings of the 31st ACM SIGACT-SIGOPS Symposium on Principles of Distributed Computing, PODC '12, New York, NY, USA, 2012. ACM. 
[12] P. Chuong, F. Ellen, and V. Ramachandran. A universal construction for wait-free transaction friendly data structures. In Proceedings of the 22nd ACM symposium on Parallelism in algorithms and architectures, SPAA '10, pages 335-344, New York, NY, USA, 2010. ACM.

[13] T. Crain, D. Imbs, and M. Raynal. Towards a universal construction for transaction-based multiprocess programs. In $I C D C N$, pages $61-75,2012$.

[14] D. Dice and N. Shavit. What Really Makes Transactions Faster? In Proc. of the 1st TRANSACT 2006 workshop, 2006.

[15] P. Fatourou and N. D. Kallimanis. The redblue adaptive universal constructions. In Proceedings of the 23rd international conference on Distributed computing, DISC'09, pages 127-141, Berlin, Heidelberg, 2009. Springer-Verlag.

[16] P. Fatourou and N. D. Kallimanis. A highly-efficient wait-free universal construction. In Proceedings of the 23nd Annual ACM Symposium on Parallel Algorithms and Architectures, pages 325 - 334, 2011.

[17] K. Fraser. Practical lock freedom. PhD thesis, Cambridge University Computer Laboratory, 2003. Also available as Technical Report UCAM-CL-TR-579.

[18] R. Guerraoui and M. Kapalka. On obstruction-free transactions. In Proceedings of the twentieth annual symposium on Parallelism in algorithms and architectures, SPAA '08, pages 304-313, New York, NY, USA, 2008. ACM.

[19] M. Herlihy. A methodology for implementing highly concurrent data structures. In Proceedings of the second ACM SIGPLAN symposium on Principles 83 Practice of Parallel Programming, PPOPP '90, pages 197-206, New York, NY, USA, 1990. ACM.

[20] M. Herlihy. Wait-free synchronization. ACM Trans. Program. Lang. Syst., 13:124-149, January 1991.

[21] M. Herlihy, V. Luchangco, P. Martin, and M. Moir. Nonblocking memory management support for dynamic-sized data structures. ACM Trans. Comput. Syst., 23:146-196, May 2005.

[22] M. Herlihy and J. E. B. Moss. Transactional memory: architectural support for lock-free data structures. In Proceedings of the 20th annual international symposium on computer architecture, ISCA '93, pages 289-300, New York, NY, USA, 1993. ACM.

[23] M. Herlihy and J. M. Wing. Linearizability: A correctness condition for concurrent objects. ACM Transactions on Programming Languages and Systems, 12(3):463-492, Jul 1990.

[24] A. Israeli and L. Rappoport. Disjoint-access-parallel implementations of strong shared memory primitives. In Proceedings of the thirteenth annual ACM symposium on Principles of distributed computing, PODC '94, pages 151-160, New York, NY, USA, 1994. ACM.

[25] V. J. Marathe, W. N. Scherer III, and M. L. Scott. Adaptive software transactional memory. In Proceedings of the 19th International Symposium on Distributed Computing, Cracow, Poland, Sep 2005. Earlier but expanded version available as TR 868, University of Rochester Computer Science Dept., May2005.

[26] D. Pelerman, R. Fan, and I. Keidar. On maintaining multiple versions in stm. In Proceedings of the 29th ACM SIGACT-SIGOPS Symposium on Principles of Distributed Computing, PODC '10, pages 16-25, New York, NY, USA, 2010. ACM.

[27] N. Shavit and D. Touitou. Software transactional memory. In Proceedings of the fourteenth annual ACM symposium on Principles of distributed computing, PODC '95, pages 204-213, New York, NY, USA, 1995. ACM. 
[28] F. Tabba, M. Moir, J. R. Goodman, A. Hay, and C. Wang. NZTM: Nonblocking zero-indirection transactional memory. In SPAA '09: Proc. 21st Symposium on Parallelism in Algorithms and Architectures, aug 2009.

[29] J. Turek, D. Shasha, and S. Prakash. Locking without blocking: making lock based concurrent data structure algorithms nonblocking. In Proceedings of the eleventh ACM SIGACT-SIGMOD-SIGART symposium on Principles of database systems, PODS '92, pages 212-222, New York, NY, USA, 1992. ACM.

[30] J.-T. Wamhoff, T. Riegel, C. Fetzer, and P. Felber. Robustm: A robust software transactional system. In S. D. et al., editor, Stabilization, Safety, and Security in Distributed Systems, Proceedings, volume 6366 of Lecture Notes in Computer Science. Springer, 2010. 


\section{A Sequential code for singly-linked list}

Figure 5 present the sequential implementation of APPEND and SEARCH. Figure 6 presents the enhanced code of Append and Search where CreateDI ReadDI and WriteDI have been incorporated in the code of Figure 5.

According to the enhanced sequential code, we have three types of operations: INITIALIzEList, APPEND, and Search. InitializeList $(\mathcal{L})$ initializes two previously declared pointers, L.start and L.end, to nil. $\operatorname{Append}(\mathcal{L}$, num $)$ appends the element num to the end of the list $\mathcal{L}$ by appending a node containing num as the next element of that pointed to by end, and updating end to point to the newly appended node. $\operatorname{SeARch}(\mathcal{L}$, num $)$ searches $\mathcal{L}$ for the first occurrence of num, starting from the element pointed to by start. SEARCH returns true if num is in $\mathcal{L}$ and false otherwise. Throughout this code, if $T$ is a type with some field $f$, then ptr to $T t$ declares that $t$ is a pointer to an object of type $T$ and $t \rightarrow f$ denotes the $f$ field of that object. CreateDi $(T)$ creates a new data item of type $T$ and returns a pointer to it. REAdDI () and WrITEDI () are used when accessing a data item or a field of a data item.

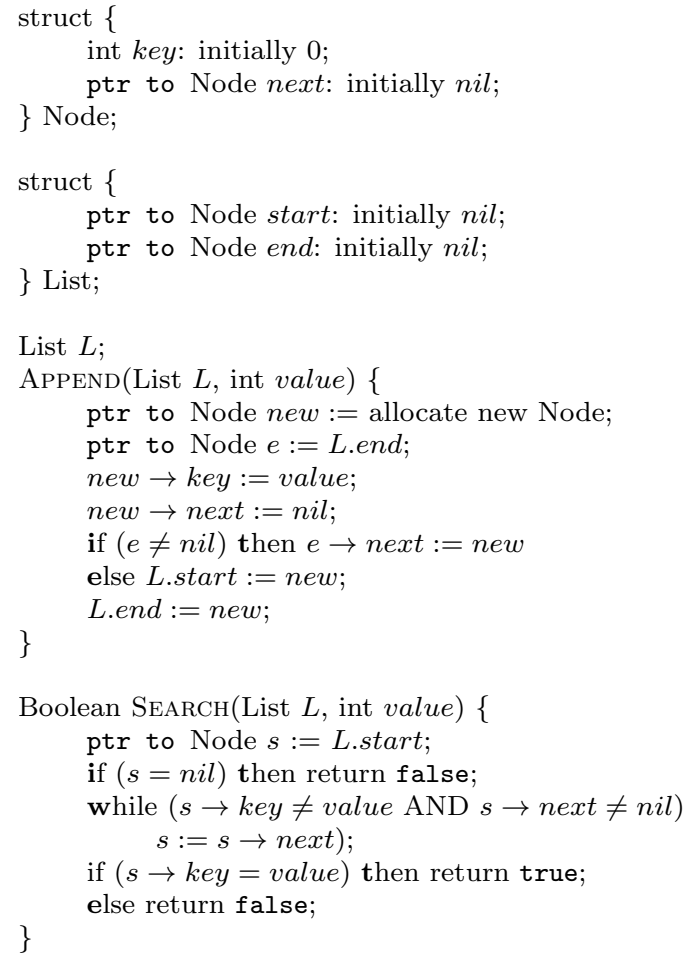

Figure 5: Sequential implementation of a singly-linked list data structure that supports APPEND and SEARCH. 


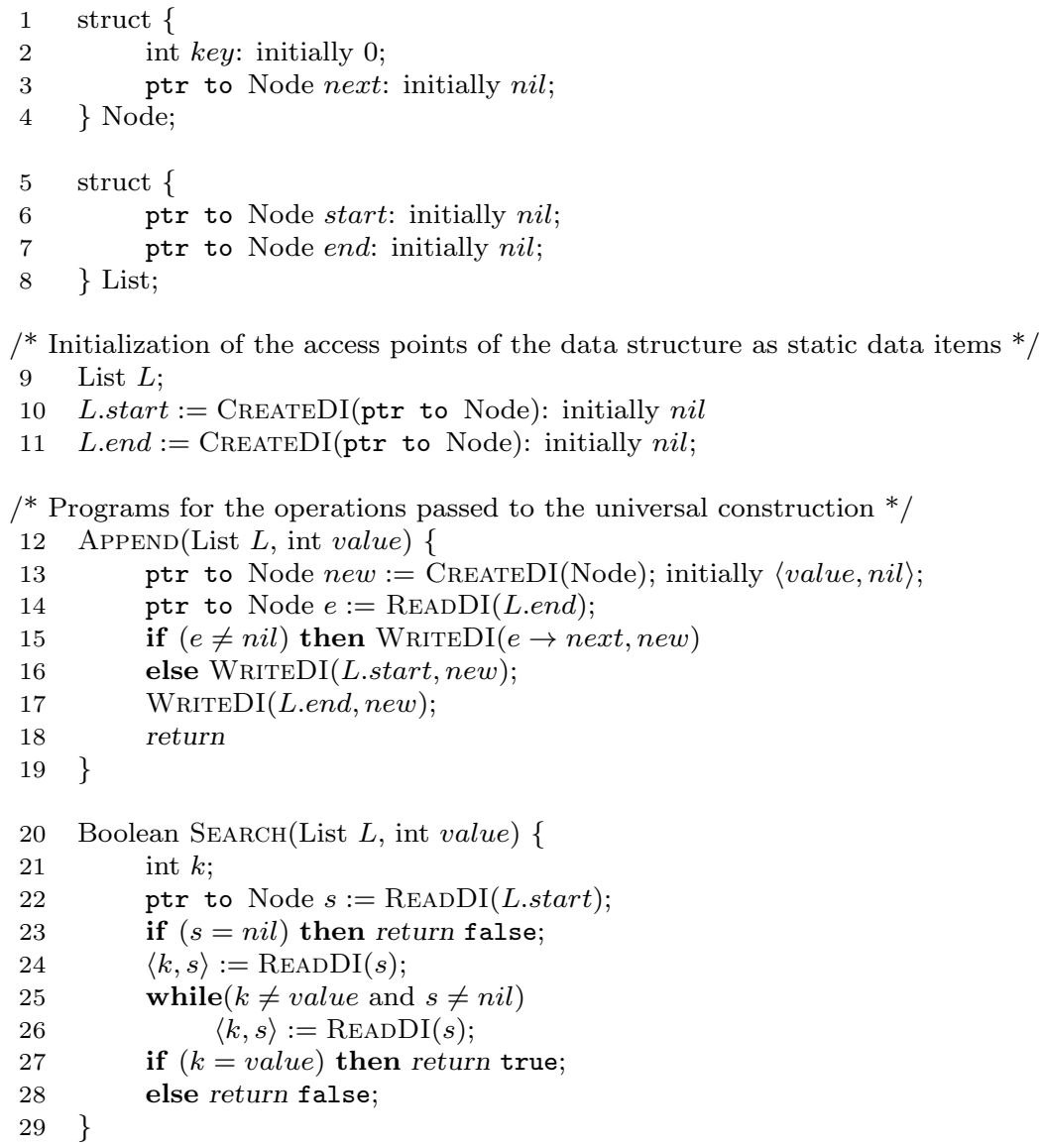

Figure 6: Enhanced version of the pseudocode of Figure 5 that includes calls to CREATEDI, READDI, and WRITEDI. 